\title{
Biologically Inspired Design and Development of a Variable Stiffness Powered Ankle-foot Prosthesis
}

DOI:

$10.1115 / 1.4043603$

Document Version

Accepted author manuscript

Link to publication record in Manchester Research Explorer

\section{Citation for published version (APA):}

Dobson, A. A., Wei, G., \& Ren, L. (2019). Biologically Inspired Design and Development of a Variable Stiffness Powered Ankle-foot Prosthesis. Journal of Mechanisms and Robotics , 11(4), [041012].

https://doi.org/10.1115/1.4043603

\section{Published in:}

Journal of Mechanisms and Robotics

\section{Citing this paper}

Please note that where the full-text provided on Manchester Research Explorer is the Author Accepted Manuscript or Proof version this may differ from the final Published version. If citing, it is advised that you check and use the publisher's definitive version.

\section{General rights}

Copyright and moral rights for the publications made accessible in the Research Explorer are retained by the authors and/or other copyright owners and it is a condition of accessing publications that users recognise and abide by the legal requirements associated with these rights.

\section{Takedown policy}

If you believe that this document breaches copyright please refer to the University of Manchester's Takedown Procedures [http://man.ac.uk/04Y6Bo] or contact uml.scholarlycommunications@manchester.ac.uk providing relevant details, so we can investigate your claim.

\section{OPEN ACCESS}




\title{
Biologically Inspired Design and Development of a Variable Stiffness Powered Ankle-foot Prosthesis
}

\author{
Alexander A Dobson \\ Research Student \\ School of Mechanical, Aerospace and Civil Bogionelewingomputing, Science and Engineering \\ The University of Manchester \\ Manchester, M13 9PL \\ United Kingdom \\ Email: alex-dobson@outlook.com \\ Guowu Wei* \\ Lecturer, Member of ASME \\ University of Salford \\ Salford, M5 4WT \\ United Kingdom \\ Email: g.wei@salford.ac.uk
}

\author{
Lei Ren* \\ Reader \\ School of Mechanical, Aerospace and Civil Engineering \\ The University of Manchester \\ Manchester, M13 9PL \\ United Kingdom \\ Email: lei.ren@manchester.ac.uk
}

\begin{abstract}
Recent advancements in powered lower limb prostheses have appeased several difficulties faced by lower limb amputees by using a Series-Elastic Actuator (SEA) to provide powered sagittal plane flexion. Unfortunately, these devices are currently unable to provide both powered sagittal plane flexion and 2-DOF at the ankle, removing the ankles capacity to invert/evert, thus severely limiting terrain adaption capabilities and user comfort. The developed 2-DOF ankle system in this paper allows both powered flexion in the sagittal plane and passive rotation in the frontal plane; a SEA emulates the biomechanics of the gastrocnemius and Achilles tendon for flexion, while a novel universal-joint system provides the 2-DOF. Several studies were undertaken to thoroughly characterize the capabilities of the device. Under both level and sloped-ground conditions, ankle torque and kinematic data was obtained by using force-plates and a motion capture system. The device was found to be fully capable of providing powered sagittal plane motion and torque very close to that of a biological ankle, while simultaneously being able to adapt to sloped terrain by undergoing frontal plane motion, thus providing 2-DOF at the ankle. These findings demonstrate that the device presented in this paper poses radical improvements to powered PAFD design..
\end{abstract}

\author{
Nomenclature \\ ABS Acrylonitrile Butadiene Styrene \\ CD Controlled Dorsiflexion \\ CFAM Custom-built Foot-Ankle Mechanism \\ COM Centre of Mass \\ COP Centre of Pressure \\ CP Controlled Plantarflexion \\ DC Direct Current \\ DOF Degree of Freedom \\ FF Foot-Flat
}

*Address all correspondence to these authors. 
FSS Forward Spring System

GRF Ground Reaction Force

HO Heel-Off

HR Heel-Rise

HS Heel-Strike

HS Heel Spring System

IC Initial Contact

IMU Inertial Measurement Unit

IEMA Inversion/Eversion Moment Arm

IMU Inertial Measurement Unit

MS Mid-Stance

SACH Solid-Ankle Cushion-Heel

SEA Series-Elastic Actuator

SW Swing

PAFD Prosthetic Ankle-Foot Device

PDMA Plantarflexion/Dorsiflexion Moment Arm

PP Powered Plantarflexion

TO Toe-Off

IR Infrared

\section{Introduction}

The ankle-foot complex plays an important role in human locomotion and the loss of a lower limb is a major disability which can pose significant detrimental effects to the amputee; limited locomotion and independence, and mental and physical health consequences [1]. Consequently, there is a great reliance on the prosthetic limb replacement to restore normal limb function and abilities to the amputee [2]. Studies indicate this heavy reliance; approximately $61 \%$ of below-knee amputees, and $88 \%$ of above-knee amputees wear their prosthesis for more than 8 hours per day [3]. As a result of this extended use of prosthetic limbs in lower limb amputees, there is a requirement for highly functional devices in order to provide a natural and comfortable experience to the user throughout their day-to-day life [1].

The use of prostheses can be traced back to the ancient Egyptians [4] and since the first prostheses created during the civilizations of Greece and Rome, different types of prosthetic replacement have been developed and among which prosthetic ankle-foot devices (PAFDs) are required by all lower limb amputees, making them the most in-demand lower limb prosthetic device. They are the primary interface between the residual limb, socket, and the ground; transferring the ground reaction force during the stance stage of the gait cycle [2]. PAFDs such as passive ankle-foot prostheses [5], quasi-passive ankle-foot prostheses [6,7], powered ankle-foot prostheses [8,9], and Bionic ankle-foot prostheses have been developed [10], gradually providing better and more comfortable lower limb prostheses for amputees based on the development of new technologies and requirement from prosthesis users. Most amputees are currently using passive or quasi-passive ankle-foot prostheses which help them with satisfactory lower-speed walking, but they continue to experience gait pathologies such as greater kinematic and kinetic leg asymmetries when walking at faster speed, higher metabolic demands and reduced self-selected walking speeds [11-14].

Modern prosthetic devices have seen a large improvement over the last several decades due to the development of strong light-weight materials, and a deeper understanding of the principles that must be abided to, to develop an effective prosthetic device. These advances have provided numerous benefits to prosthetic devices, such as improving the comfort, function, and cosmetics, all of which have contributed significantly to lower limb amputees gaining improved performance and physical capabilities [2]. Consequently, there is a growing number of amputees choosing to use prosthetic devices to restore function, resulting in increasing market demands for both quantity and quality of lower limb prosthetic devices. According to such demands, the powered and bionic ankle-foot prostheses are proposed, designed and developed. It is expect that the powered ankle-foot prostheses can generate positive net work during the stance phase and biological levels of mechanical power during terminal stance [15]. Research on powered ankle-foot prosthesis was proposed in the later 1990s; attempting to develop better prosthesis to improve the locomotion of amputees. Since this concept being proposed, a great deal of research work has been done on the study of using amputee's residual limb EMG signals to control the ankle position of an active ankle-foot prosthesis [16]. These include the investigation of an ankle-foot emulator system for the understanding of human walking biomechanics [17]; the development of the MIT powered ankle-foot prosthesis for improving the amputee ambulation [18]; the implementation of a myoelectric-driven, finite state controller to a powered ankle-foot prosthesis for producing net propulsive work during level-ground walking and performing shock absorption during stair descent [19]; the exploration of using powered ankle-foot prosthesis to improve walking metabolic economy [20]; the 
examination of evaluation of slope-walking by the gait experiments [21]; the neuromuscular-model-based adaptive musclereflex controller for improving adaptation to environment disturbances across varied terrain surfaces [8]; and the comparative study on the effect of an ankle-foot prosthesis on kinetic loading of the unaffected leg during level-ground walking, revealing the advantages of using a powered ankle-foot prosthesis [9].

Further, ankle-foot prosthesis can be of Solid-Ankle Cushion-Heel (SACH), single-axis, and multi-axis [22] and most of the current powered ankle-food prostheses are of single-axis due to the infancy of the powered PAFD technology; hence their many benefits also come with a critical drawback, i.e. the single degree-of-freedom prosthesis only provide plantarflexion in the sagittal plane but not dorsiflexion; leading to the low terrain adaption and reduced user comfort. Therefore, because of the aforementioned importance and demand of lower limb prosthetic development, and the benefits of powered PAFDs and their current limitations, this paper aims to further such progress with focus on the design and development of a biologically inspired powered PAFD that is capable of providing 2-DOF motion at the ankle. Mechanical design of a 2-DOF powered ankle-foot prosthesis is presented, and a prototype of the proposed prosthesis was developed. Further, manual-operation based test was implemented; leading to the verification of the proposed concept and feasibility of the 2-DOF powered anklefoot prosthesis laying background for further investigation and improvement.

\section{Mechanical Design of a Stiffness Variable PAFD}

There are a series of design goals and specifications which will be aimed for during the development of the PAFD. These specifications exist in the hope that the final PAFD will be able to effectively mimic the mechanics of its biological counterpart. Firstly, to provide terrain adaption and increased user comfort, the prosthetic ankle must provide 2-DOF; rotation in the sagittal plane (plantarflexion/dorsiflexion), and rotation in the frontal plane (inversion/eversion). This 2-DOF ankle must also allow for powered sagittal plane flexion and adaption to (inverted/everted) sloped-ground conditions in which the direction of walking is perpendicular to that of the direction of the ground's slope. Secondly, the device must be able to control the absolute position of the ankle during the Controlled Plantarflexion (CP), Controlled Dorsiflexion (CD), Powered Plantarflexion (PP), and Swing (SW) sub-phases, and thus must have the ability to determine key gait events, such as HeelStrike (HS), Foot-Flat (FF), Heel-Rise (HR), and Toe-Off (TO). Thirdly, to provide a reduction to the metabolic rate of the user, the device must provide positive net work during locomotion, and, if sufficient stored energy is not readily available, the device must be able to act as a passive device. Finally, for inertial symmetry between both lower limbs during locomotion, the device must weigh approximately the same as the amputated biological limb of equivalent height.

\subsection{Identification of Structural Parameters}

By analysing several parameters of the human ankle during locomotion, the design specifications and parameters required to meet the aforementioned aims are as follows. 1) Degrees of freedom: during walking, the ankle allows approximately $10^{\circ}-15^{\circ}$, and $20^{\circ}-25^{\circ}$ of dorsiflexion and plantarflexion respectively. During level-ground walking, the ankle does not rotate significantly in the frontal plane, although, as to provide terrain adaption to inverted/everted sloped-ground surfaces, it is necessary that the ankle is allowed to rotate with a range close to that of the biological counterpart; $20^{\circ}-30^{\circ}$, and $10^{\circ}-15^{\circ}$ of inversion and eversion respectively [7]. 2) Total height: for a human of $1.75 \mathrm{~m}$ tall, the average distance between the knee and sole of the foot is $0.5 \mathrm{~m}$ [8], and the average height of transtibial amputation is approximately two thirds of this height, measured from the sole upward [9]. Therefore, the height of the device should not exceed $\sim 0.33 \mathrm{~m}$. 3) Foot length: the average length of a male's foot is approximately $0.268 \mathrm{~m}$ [9]. 4) Total mass: for a $1.75 \mathrm{~m}$ tall person, with a mass of $75 \mathrm{~kg}$, the mass of the lower limb of height $0.33 \mathrm{~m}$, measured from the sole upward, is approximately 2.8kg. 5) Torque: a peak torque of $\sim 1.28 \mathrm{Nm} / \mathrm{kg}$ is reached at the start of PP [10]. 6) Energy: according to [6], for a person walking at $1.25 \mathrm{~m} / \mathrm{s}$ under level-ground conditions, the approximate net work done at the ankle is $\sim 0.13 \mathrm{~J} / \mathrm{kg}$. For convenience, the design specifications and limits are listed in Table 1.

\subsection{Structural Design and Analysis}

Based on the design specifications and structural parameters identified in Section 2.1, a powered prosthetic ankle-foot device (PAFD) is designed in this paper as shown in Fig. 1. The proposed PAFD contains the ankle unit and the food unit. The ankle unit is made up of several parts, fashioning as a custom Universal-joint (U-joint). The cross of the U-joint differs from those commonly used, in that the cross axle normal to the frontal plane is extended out of the joint at both sides, acting as a Plantarflexion/Dorsiflexion Moment Arm (PDMA) for both sagittal plane spring systems. Similarly, the cross axle normal to the sagittal plane acts as an Inversion/Eversion Moment Arm (IEMA) for the spring system located within the ankle U-joint in the frontal plane. The top half of the ankle U-joint is fixed to the shank. The shank provides the structural to which other components are constrained.

There are three spring systems, two located on either side of the ankle U-joint in the sagittal plane, and one contained within the ankle U-joint in the frontal plane. The spring system located at the heel side of the ankle U-joint in the sagittal plane - Heel Spring System (HSS) - is connected in series with a linear actuator. This linear combination of spring system 
Table 1. Design specifications and parameters that will be aimed for.

\begin{tabular}{lc}
\hline Specification & Target value \\
\hline Total height $(\mathrm{m})$ & 0.33 \\
\hline Foot length $(\mathrm{m})$ & 0.26 \\
\hline Total device mass $(\mathrm{kg})$ & 2.8 \\
\hline Max. Ankle torque $\left(\mathrm{Nmkg}^{-1}\right)$ & 1.28 \\
\hline Cycle energy $\left(\mathrm{Jkg}^{-1}\right)$ & 0.13 \\
\hline Max. Planterflexion angle $\left(^{\circ}\right)$ & 25 \\
\hline Max. Dorsiflexion angle $\left(^{\circ}\right)$ & 15 \\
\hline Max. Inversion angle $\left(^{\circ}\right)$ & 20 \\
\hline Max. Eversion angle $\left(^{\circ}\right)$ & 15 \\
\hline
\end{tabular}

and actuator forms a Series-Elastic Actuator (SEA). The bottom spring end of the SEA is fixed to the end of the heel PDMA, and the top end of the actuator is fixed to the shank. The spring system located at the forward side of the ankle in the sagittal plane - Forward Spring System (FSS) - is fixed at the bottom end to the end of the forward PDMA, and the top end is free to slide along a slot constrained at the shank. The spring system located within the ankle U-joint in the frontal plane - Frontal Parallel Spring (FPS) system - consists of two identical springs which make up a parallel spring system. The top end of each of these FPSs are secured to their respective IEMA (left or right), and the bottom end of each is constrained to the base half of the ankle U-joint. The base half of the ankle U-joint is fixed to the prosthetic foot unit. The foot is of simple design, following similar general shape to that of a biological foot. Figure 2 illustrates a kinematic diagram for the PAFD, noting major mechanisms and motion.

\subsubsection{Desired Gait and Motion.}

In this paper, the motion of the PAFD is designed to best emulate that of a biological ankle during a normal level-ground and inverted/everted sloped-ground walking gait. The motion of the PAFD during both of these ground conditions is similar, differing only by the inversion/eversion which is experienced under the sloped-ground conditions. By evaluating gait cycle events, the motion of the PAFD at series of time frames was generated using SolidWorks. Figure 3(a) shows the intended sagittal plane motion of the PAFD under both level and sloped-ground conditions, and Fig. 3(b) shows the intended frontal plane motion under sloped-ground conditions.

\subsubsection{Ankle Design.}

The ankle is the fundamental mechanism to the PAFD. Comprising of several sub-components, the ankle allows for 2-DOF with powered and passive motion permitted in the sagittal plane, and passive motion permitted in the frontal plane. There are four main sub-components of the ankle: Series-Elastic Actuator (SEA), Forward Spring System (FSS), Frontal Parallel Spring (FPS) system, and the ankle U-joint itself. The SEA and FSS provide torque in the sagittal plane, whereas the FPS system provides torque in the frontal plane. The overall aim with the ankle design was to produce an ankle which would provide the most natural gait during locomotion, in terms of both kinematics and torque.

\subsubsection{Series-Elastic Actuator and Forward Spring System.}

Sagittal plane motion of the ankle includes plantarflexion and dorsiflexion, occurrence of which is enabled by the gastrocnemius and tibialis anterior respectively [23]. By utilising the sagittal plane mechanical characterisations of these muscles established in [24], two systems were developed to emulate the necessary motion and torque which experienced at the ankle during a normal level-ground gait cycle. These systems are the SEA and FSS.

The SEA and FSS are the two systems which provide plantarflexion. Design of the FSS was simple; springs of sufficiently high stiffness are connected in parallel, constrained to the sagittal plane by the end of the forward PDMA and slider, and allowed to only undergo compression by the use of the slider. For the SEA, on the other hand, more was considered. As the SEA is made up of a spring and linear actuator connected in series, optimal actuator displacement during a gait cycle must also be determined. The actuator displacement shown in Fig. 4 was chosen with several considerations. Firstly, the expected actuator motion must be feasible to achieve the available resources. Secondly, actuator motion should provide as natural motion as possible. Finally, actuator motion should be efficient by reducing extension/contraction speed if possible. 


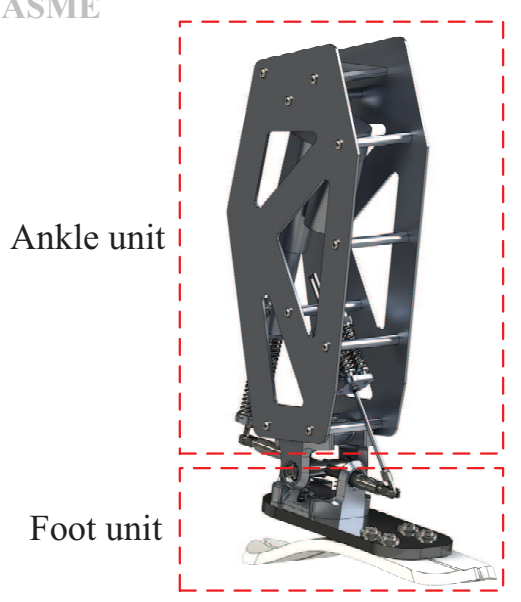

(a)

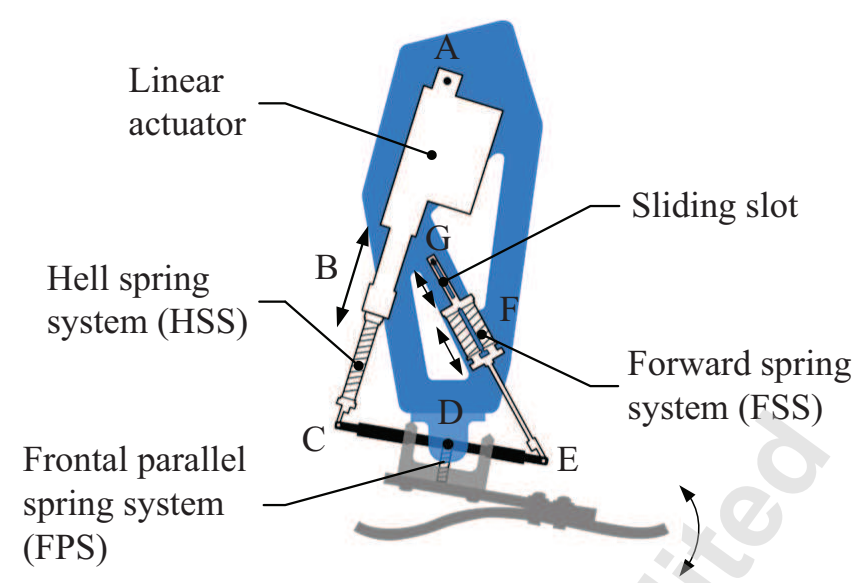

(b)

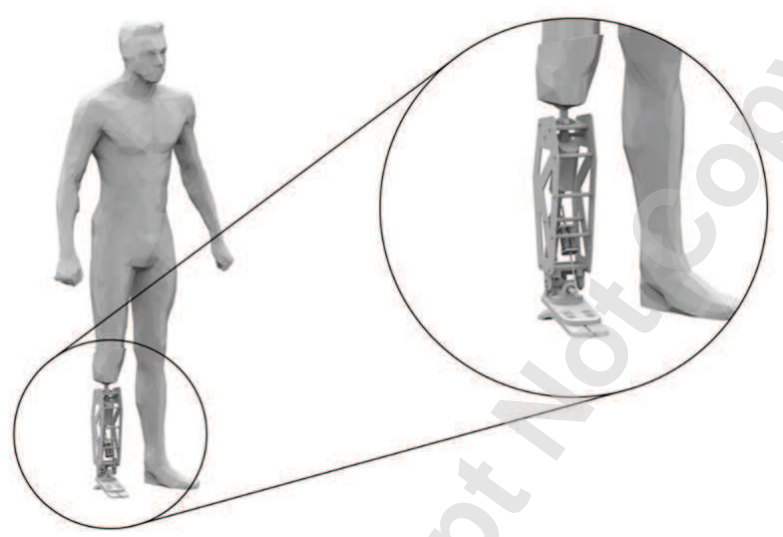

(c)

Fig. 1. (a) CAD render of the final PAFD design, (b) Sagittal plane cut-out diagram, and (b) CAD render of the PAFD being worn by a user.

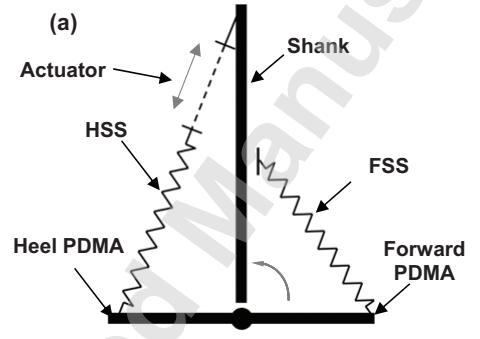

(b)

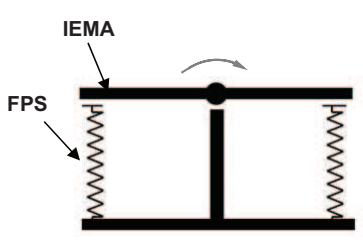

Fig. 2. Kinematic diagram of the PAFDs: (a) Sagittal plane, and (b) Frontal plane.

\subsubsection{Frontal Parallel Spring System.}

Under smooth or rough sloped-ground conditions the ankle will undergo either inversion or eversion, depending on the direction of slope. Additionally, under level-ground conditions, the ankle will also undergo significant amount of inversion and eversion [26]. In addition to providing sufficient resistance to this motion, the ankle has been designed to provide an adequate range of motion under these conditions. Therefore, to emulate the muscles and tendons on the medial and lateral sides of the ankle's frontal rotational axis which provide inversion and eversion respectively [27] a novel passive parallel spring system has been developed in an attempt to provide the aforementioned range of motion.

\section{Prototype and Finite State Controller}

\subsection{Prototype of the PAFD}

Figure 5 indicates the CAD model and final fully-assembled PAFD. The ankle joint was designed to permit the specified amount of powered sagittal plane motion and passive frontal plane motion. As no off-the-shelf U-joint was able to meet the 
(a)

(1)

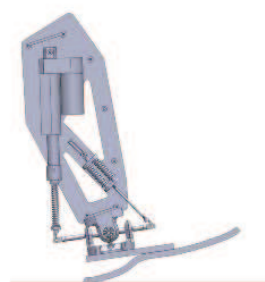

(2)

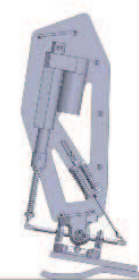

(3)

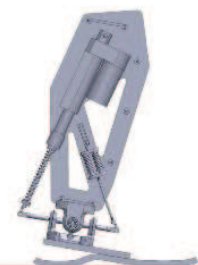

(4)

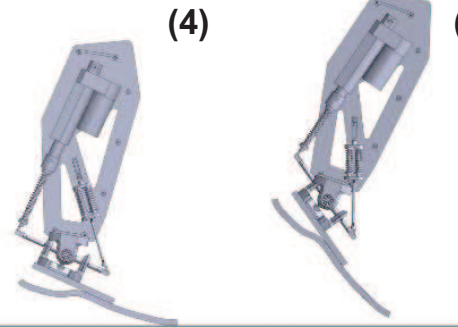

(5)

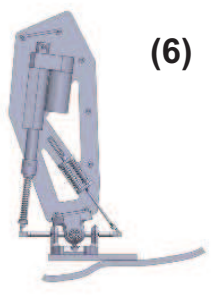

(6) (b) (1)

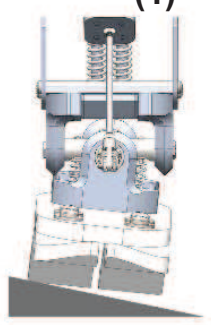

(2)

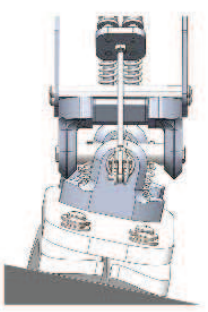

(3)

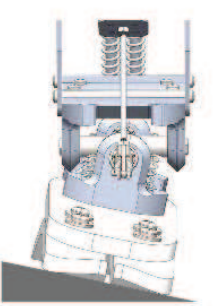

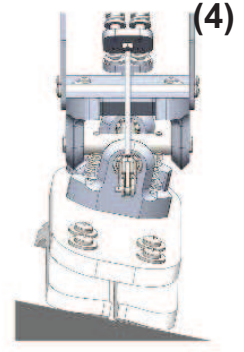

(4)

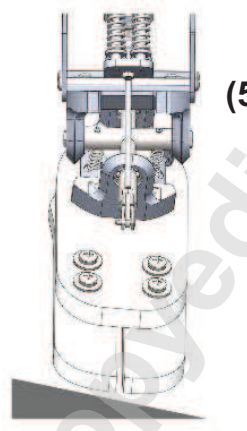

Fig. 3. The intended motion of the PAFD in the sagittal (a) and frontal (b) planes. The key gait events, periods, and sub-phases represented are: (1) HS, (2) CD, (3) HO, (4) PP, (5) TO, and (6) SW.

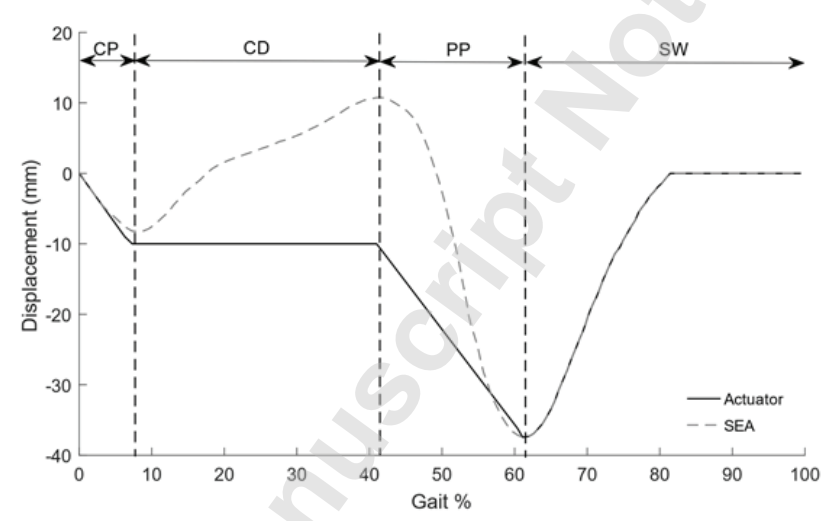

Fig. 4. Desired displacement of the actuator, and SEA. The SEA displacement data was generated using desired ankle angle data from [1]; graph plotted in the style of [25].

necessary mechanics required by the ankle joint, a custom ankle joint was designed, as seen in Fig. 6 . The two halves of the ankle U-joint (top and base), are near identical, though the base half contains two slots to house the base of each FPS. The overall shape of each U-joint half was chosen so that any amount of powered sagittal plane flexion may be permitted, at any amount of frontal plane rotation, and vice versa.

To provide the two moment arms for each FPS (IEMAs), the ankle U-joint axle normal to the sagittal plane contains two slots which house the top of each FPS (akin to the U-joint base) (see Fig. 6). To provide the two moment arms for the two sagittal plane spring systems (PDMAs), two lever-like components are screwed onto the ends of the U-joint axle which runs normal to the frontal plane as shown in Fig. 6.

The HSS is a simple mechanism. Two springs are used in order to provide the necessary stiffness. Each spring surrounds a piston like component which is used to ensure that each spring does not undergo buckling when compressed. So that each spring can undergo both extension and compression, the ends of each spring is welded to their respective half of their piston. At the top end of the HSS is a housing which is used to secure the actuator arm. At the bottom end of the HSS is flat-eye which is used to secure the HSS to the end of the heel PDMA.

The FSS is very similar to the HSS, with the spring mechanism being near identical, but instead of two springs, there is one. At the top end of the FSS is a slider which is required so that the FSS may only undergo compression, otherwise it would resist plantarflexion at ankle angles $\theta<0^{\circ}$. At the bottom end of the FSS is flat-eye which is used to secure the FSS to the forward PDMA. 

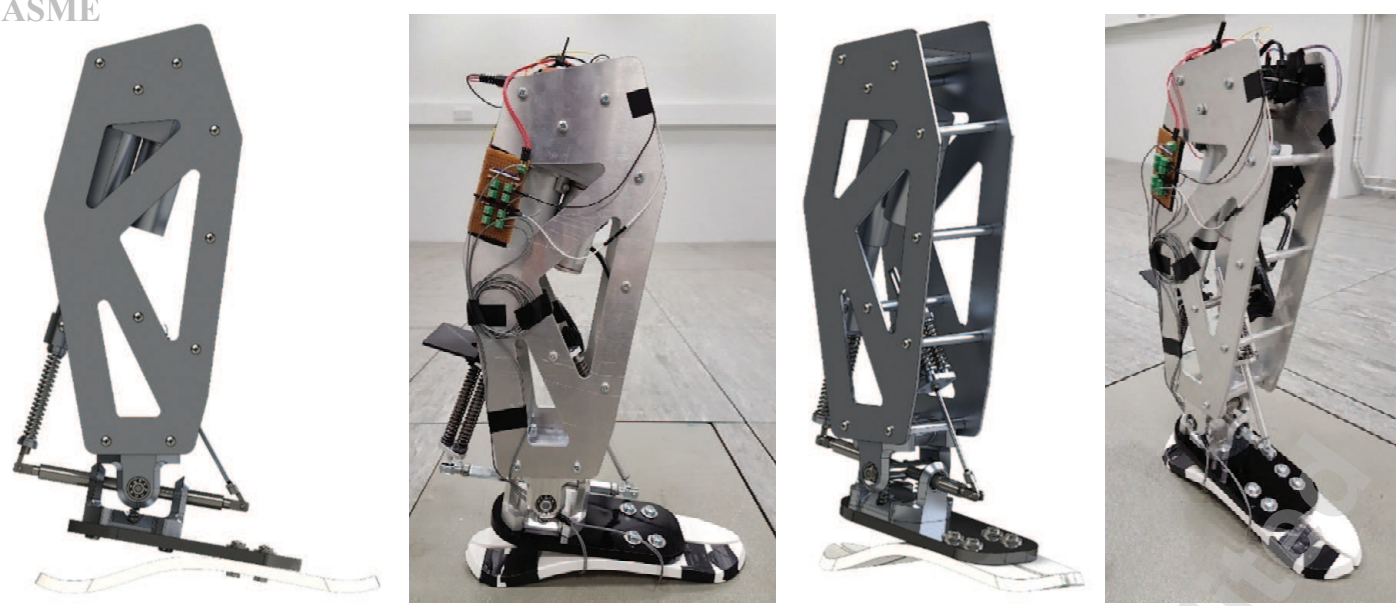

Fig. 5. CAD render and photographs of the PAFD (including control system).
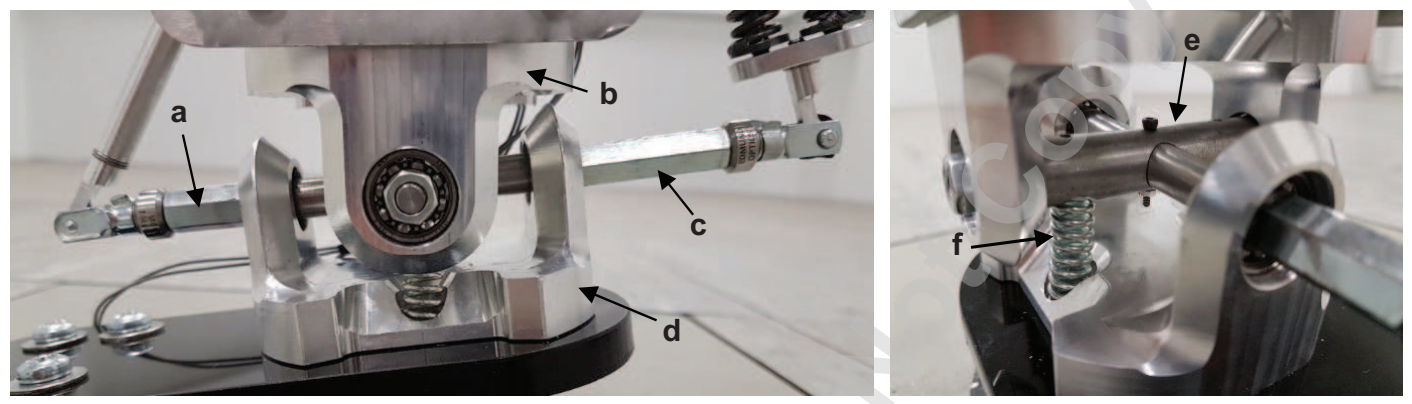

Fig. 6. Close up of the U-joint ankle mechanism: a) Forward PDMA, b) U-joint Top, c) Heel PDMA, d) U-joint Base, e) IEMA, and f) FPS.
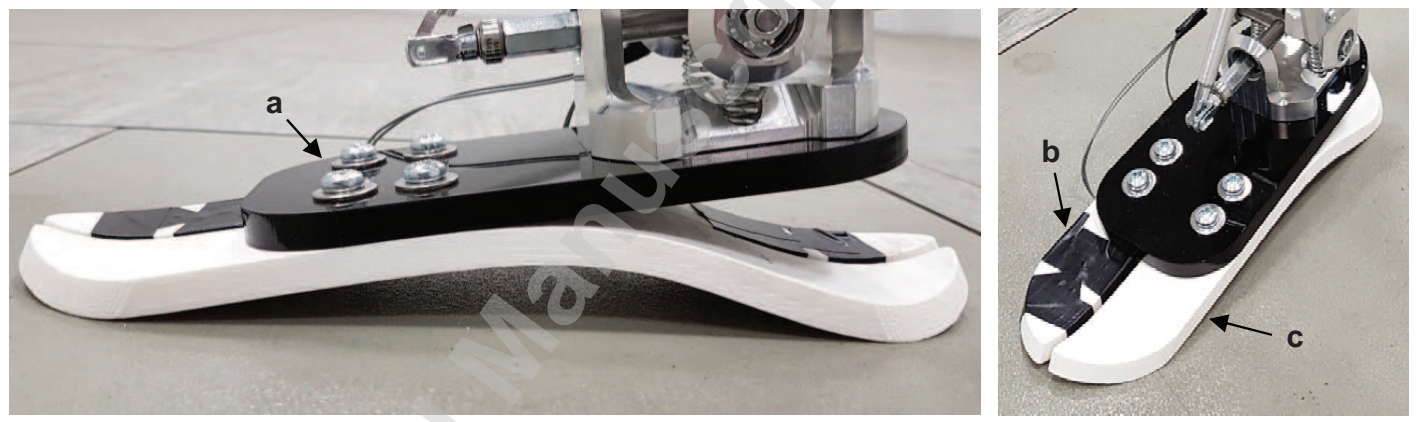

Fig. 7. The assembled prosthetic foot: a) Ankle platform, b) Right stabilizer, and c) Left stabilizer.

The top and base of the ankle U-joint are directly bolted to the shank and foot respectively (see Fig. 5). The FSS slot is constrained to one of the shanks' pylons, and the HSS housing is secured to the linear actuator which is also constrained to one of the shanks pylons as indicated in Fig. 5.

For the ankle U-joint components, the materials used are steel and aluminium. Where possible, aluminium was preferred due to its reasonably high strength and low density, but for certain components which undergo particularly high amounts of stress, such as the U-joint cross, steel is required.

The prosthetic foot was designed such that it could deform/bend under the cyclic loading of locomotion, with the intention of providing adequate comfort to the user, and prevent potential damage to the devices mechanisms due to shock. This nature can be provided by the choice of shape and materials of the foot. To achieve the shape of the foot, inspiration has been drawn from study of the human foot, in addition to researching current prosthetic foot designs. The assembled prosthetic foot is presented in Fig. 7. The foot consists of three main components, i.e. left and right stabilizers, and the ankle platform. The left and right stabilizers are secured to the ankle platform using two screws each. These two screws restrict all motion of the stabilizers relative to the ankle platform. Similar to the ankle, connection of the foot to the rest of the PAFD is simple; the ankle platform is screwed directly the ankle U-joint base. 
Table 2. Desired design specifications and what was finally achieved in the prototype

\begin{tabular}{lcc}
\hline Specification & Target value & Achieved value \\
\hline Total height $(\mathrm{m})$ & 0.33 & 0.43 \\
\hline Foot length $(\mathrm{m})$ & 0.26 & 0.28 \\
\hline Total device mass $(\mathrm{kg})$ & 2.8 & 2.95 \\
\hline Max. Ankle torque $\left(\mathrm{Nmkg}^{-1}\right)$ & 1.28 & 1.40 \\
\hline Cycle energy $\left(\mathrm{Jkg}^{-1}\right)$ & 0.13 & 0.28 \\
\hline Max. Planterflexion angle $\left(^{\circ}\right)$ & 25 & 22 \\
\hline Max. Dorsiflexion angle $\left(^{\circ}\right)$ & 15 & 18 \\
\hline Max. Inversion angle $\left(^{\circ}\right)$ & 20 & 20 \\
\hline Max. Eversion angle $\left(^{\circ}\right)$ & 15 & 20
\end{tabular}

The foot was constructed from a combination of 3D printed ABS and laser cut Perspex. The left and right stabilizers are made from ABS, while the ankle platform is made from Perspex. ABS was chosen for the stabilizers due to its high toughness and reasonable flexibility, allowing the heel to bend at HS, thus providing shock absorption. Perspex was chosen over ABS for the ankle platform due to its greater strength, a property which is required by the ankle platform due to large moments it experiences. Table 2 presents a comparison between the design specifications detailed in Section 2 and the final parameters achieved in the prototype.

The function of the control system is to regulate the motion of the linear actuator according to the intended motion of the PAFD as specified in Section 2.2.1. Hence, a finite state controller was programmed in C, and run in real-time using an Arduino Uno mounted on-board the PAFD. The finite state controller utilizes data from several sources to determine the gait events and phases which have occurred and are currently underway, in addition to the current actuator length. The sensors utilized by the control system provide the current pressure at the toe and heel end of the prosthetic foot, and the current length of the linear actuator.

\subsection{Finite State Controller}

Each gait sub-phase, CP, CD, PP, and SW are initiated by a single gait event, HS, FF, HO, and TO respectively. Thus, the occurrence of these events may be used to determine the current gait sub-phase which is underway. Each gait event, HS, $\mathrm{FF}, \mathrm{HO}$, and $\mathrm{TO}$ are unique in the sense that each are associated with their own combination of heel and toe contact with the ground. By taking advantage of these associations the finite state controller takes several inputs and determines the gait sub-phase which is currently underway. The finite state controller makes uses of three classification trees, each of which classify sensor data into current heel and toe states, the current gait event, and the current gait cub-phase, as shown in Fig. 8.

For the toe and heel sensors, two strain gauges are utilized, one placed at the toe and one at the heel of the prosthetic sole, as shown in Fig. 9.

In addition to determining the current gait sub-phase, the necessity to enable or disable the linear actuator, and control its direction, must also be determined by the finite state controller. A simple method would be to follow the actuator path presented in Fig. 4. There are several issues with this method due to its reliance on the pre-defined duration of gait sub-phases, and consequently a system is required where the current actuator extension/contraction is also taken into consideration. For the actuator sensor, an infrared range sensor is utilized. This sensor is mounted to the base of the linear actuator, with a reflective panel fixed to the actuator piston, as seen in Fig. 10. The IR sensor then measures the distance from its position on the actuator base to the position of the reflective panel on the piston, providing the actuator length, $L_{a c t}$.

\section{Manual-manipulation Based Experiment and Data Representation}

\subsection{Experimental Protocol}

As the primary purpose of this paper is to develop a powered 2-DOF PAFD, two sessions were undertaken to fully investigate the PAFD's 2-DOF capabilities: 1) Level-Ground Session, and 2) Sloped-Ground Session. Additionally, although not a primary design goal, the ability for the device to also act passively was a design aim and consideration. Therefore, to test the PAFDs powered and passive capabilities, the two sessions were further broken down into two studies: A) Powered 

more: I) with FSS Study, and II) without FSS Study.

Overall, a total of eight studies were undertaken: (1A-I) Level-Ground and Powered with FSS; (1A-II) Level-Ground and Powered without FSS; (1B-I) Level-Ground and Passive with FSS; (1B - II) Level-Ground and Passive without FSS; (2A-I) Sloped-Ground and Powered with FSS; (2A - II) Sloped-Ground and Powered without FSS; (2B-I) Sloped-Ground and Passive with FSS; (2B-II) Sloped-Ground and Passive without FSS. All studies measured the ankle torque in the sagittal plane, and PAFD kinematics during a walking gait cycle. To obtain this data, each study involved several walking trials, and made use of force-plates and a motion capture system. The PAFD was manually walked by an operator in a manner which closely emulated the motion of the lower limb during a walking gait cycle. Figure 11 shows the setup used to emulate the motion of the lower limb during a walking gait cycle. This setup was used for all studies, though the process employed by the operator differed between the powered and passive studies.

For both the Powered Level-Ground and Sloped-Ground Studies (1A and 2A), the general process is conducted as: 1) The PAFD is lowered to the ground surface for HS. 2) During CP, the ankle is allowed to freely plantarflex until FF. 3) At FF, a downforce is applied by the operator leaning forward and supporting their upper body weight with the PAFD. To provide the correct motion during $\mathrm{CD}$, the operator leans further forward, rolling over their knees as though they act as pivots. 4) By estimating the point when the correct amount of dorsiflexion had been achieved during $\operatorname{CD}\left(\theta=10^{\circ}\right)$, the PAFD is then tilted in such a way to cause the heel to lift from the ground (HO), thus beginning PP. 5) During PP, the PAFD continues to support the operators upper body weight, in addition to pushing them upwards as to emulate the additional force required to propel the user forward during locomotion. 6) When maximum plantarflexion has occurred at the end of PP, the PAFD is lifted from the ground to begin SW. 7) During SW, the ankle is allowed to return to its default angle, completing a single gait cycle.

For both the Passive Level-Ground and Sloped-Ground Studies (1B and 2B), the process followed is: 1) The PAFD is lowered to the ground for HS. 2) As in passive mode the PAFD is unable to plantarflex past the default ankle angle, downforce is applied at the instant of HS. This is achieved by the operator leaning forward and supporting their upper body weight with the PAFD. To provide the correct motion during CD, the operator leans further forward, rolling over their knees as though they act as pivots. 3) By estimating the point when the correct amount of dorsiflexion had been achieved during $\mathrm{CD}\left(\theta=10^{\circ}\right)$, the PAFD is then tilted in such a way to cause the heel to lift from the ground (HO), thus beginning PP. 4) As in passive mode the PAFD is unable to plantarflex past the default ankle angle, at the instant of HO, the PAFD is lifted from the ground. 5) During SW, the ankle is allowed to return to its default angle, completing a single gait cycle.

Conditions during each trial were similar and repeatable, rather than the operator applying the downforce using their arm muscles, operator applied his body-weight.

\subsection{Motion Capture System}

PAFD kinematics were obtained by using a Vicon motion capture system. This motion capture system made use of six IR cameras, which recorded the positional data of a series of IR markers positioned at various locations on the PAFD. The locations of these IR markers were chosen such that, not only the kinematics of the PAFD could be measured, but also the ankle torques, as the position and direction of the 2-DOF ankles two axles is required for the resolving of the moments and forces about them. Figure 12 shows the PAFD with the IR reflectors in position.

During studies 1A and 1B, as illustrated in Fig. 13, the PAFD undertook several walking gait cycle trials under levelground conditions. During each trial, the PAFD was walked over a force-plate under level-ground conditions. For studies $2 \mathrm{~A}$ and $2 \mathrm{~B}$, an inverted slope with an angle of $20^{\circ}$ was positioned onto the force-plate (see Fig. 13(b)). The PAFD was then walked across the slope, perpendicular to the direction of the slope under sloped-ground conditions.

During studies 1A and 2A only, the actuator was connected to a $24 \mathrm{~V}$ DC bench power supply and acted in a powered manner. Additionally, although an on-board power supply is included for the microcontroller ( $9 \mathrm{~V}$ battery), for debugging and troubleshooting purposes, the Arduino was connected to a computer during these studies. The overall equipment setup for studies $1 \mathrm{~A}$ and $2 \mathrm{~A}$ is presented in Fig. 13(c).

\subsection{Position, Angle and Force Representation}

The post-processing of experimental data was an extensive process, requiring manual inspection and sorting of large data sets. The ankle angle in both the sagittal and frontal planes, ankle torque in the sagittal plane, and net PAFD energy expenditure was calculated for each trial.

Ankle angle calculations were achieved by using the motion capture data of the several IR marker locations throughout each trial. The shank, ankle, and toe allowed for the calculation of four vectors shown in Fig. 14: (1) the shank vector, $\boldsymbol{s}=\overrightarrow{K L} ;(2)$ the ankle vector normal to the sagittal plane, $\boldsymbol{a}_{2}=\overrightarrow{K E} ;(3)$ the ankle vector normal to the frontal plane, $\boldsymbol{a}_{1}=\overrightarrow{K G}$; and (4) the foot vector, $f=\overrightarrow{B A}$. The ankle angle in the sagittal plane was determined by calculating the angle between vectors 


$$
\theta=\theta_{0}-\arccos \left(\frac{\boldsymbol{s} \cdot \boldsymbol{a}_{1}}{\|\boldsymbol{s}\|\left\|\boldsymbol{a}_{1}\right\|}\right)
$$

and the ankle angle in the frontal plane was determined by calculating the angle between vectors $f$ and $\boldsymbol{a}_{2}$ :

$$
\theta_{F}=\arccos \left(\frac{\boldsymbol{f} \cdot \boldsymbol{a}_{2}}{\|\boldsymbol{f}\|\left\|\boldsymbol{a}_{2}\right\|}\right)
$$

Using the force-plate data and calculated PAFD vectors, the ankle torque in the sagittal plane was calculated. The ankle torque in sagittal plane is given as the torque about the ankle vector normal to the sagittal plane as

$$
T=(\boldsymbol{F} \times \boldsymbol{r}) \cdot \boldsymbol{a}_{2}
$$

Where $\boldsymbol{F}$ is the reaction force vector measured by the force-plate, and $\boldsymbol{r}=\overrightarrow{M K}$, the vector starting at the COP and ending at the centre of the ankle U-joint where the two axles cross.

Once ankle angle and torque had been calculated for each gait cycle, the PAFD net work could be determined. The net work is given as the product of ankle torque and angle in the sagittal plane throughout each gait cycle and ignores the negligibly small amount of frontal plane work done.

$$
W=T \times \theta
$$

Ankle torque and net work has been normalized for the sake of comparison with the target PAFD performance. Due to the methods employed to test the PAFD, assumptions also had to be made regarding the normalising of the data per unit mass of the user's weight. Thus, a relation between the vertical Ground Reaction Force (GRF) and the user's mass is assumed; the user's weight is approximately equal to the peak vertical GRF [6,10]. Hence, the effective user mass for a given trial is given by:

$$
m_{e}=\frac{F_{G R F} \underline{k}}{g}
$$

Where $m_{e}$ is the effective user mass, and $F_{G R F} \underline{k}$ is the peak of the GRF's vertical component during for a given trial. The normalised ankle torque and net work are thus given by Eqs. (6) and (7) below:

$$
t=\frac{T}{m_{e}}
$$

and

$$
w=\frac{W}{m_{e}}
$$

\section{Results and Discussions}

\subsection{Ankle Angle}

\subsubsection{Level Ground.}

The sagittal plane ankle angle during a single trial for the PAFD in powered and passive modes, both with and without the FSS, and under level-ground conditions (studies 1A and 1B) are presented in Fig. 15. Mean maximums of these values across several trials are also presented in Table 3 . 
Copyright Takla 13. bSurninaly of ankle angle measurements taken from several trials for Session 1. Max. Inversion/Eversion measurements are not presented for Session 1 as all studies are under level-ground conditions, and thus negligible amounts of inversion/eversion are undergone.

\begin{tabular}{lccc}
\hline Study & Max. plantarflexion angle $\left(^{\circ}\right) \pm$ SD & Max. dorsiflexion angle $\left(^{\circ}\right) \pm$ SD & Max. inversion/eversion angle $\left(^{\circ}\right) \pm$ SD \\
\hline 1A-I & $-16.27 \pm 1.95$ & $1.879 \pm 1.05$ & - \\
\hline 1A-II & $-13.62 \pm 3.89$ & $3.091 \pm 1.66$ & - \\
\hline 1B-I & $-0.34 \pm 0.51$ & $5.038 \pm 1.18$ & - \\
\hline 1B-II & $-1.16 \pm 0.73$ & $9.452 \pm 1.41$ & - \\
\hline
\end{tabular}

Table 4. Summary of ankle angle measurements taken from several trials for all Session 2

\begin{tabular}{lccc}
\hline Study & Max. plantarflexion angle $\left(^{\circ}\right) \pm \mathrm{SD}$ & Max. dorsiflexion angle $\left(^{\circ} \pm \mathrm{SD}\right.$ & Max. inversion/eversion angle $\left(^{\circ}\right) \pm \mathrm{SD}$ \\
\hline 2A-I & $-13.43 \pm 2.49$ & $2.318 \pm 1.46$ & $20.85 \pm 2.60$ \\
\hline 2A-II & $-14.47 \pm 3.69$ & $3.542 \pm 2.58$ & $20.45 \pm 1.90$ \\
\hline 2B-I & $-0.02 \pm 0.68$ & $5.879 \pm 1.22$ & $19.98 \pm 2.21$ \\
\hline 2B-II & $-1.70 \pm 1.03$ & $10.25 \pm 1.61$ & $19.08 \pm 2.14$ \\
\hline
\end{tabular}

Table 5. Summary of ankle torque measurements taken from several trials for Session 1. Max. Inversion/Eversion measurements are not presented for Session 1 all studies are under level-ground conditions, and thus no inversion/eversion is undergone.

\begin{tabular}{lcc}
\hline Study & Max. plantarflexion torque $(\mathrm{Nm} / \mathrm{kg}) \pm \mathrm{SD}$ & Max. dorsiflexion torque $(\mathrm{Nm} / \mathrm{kg}) \pm \mathrm{SD}$ \\
\hline 1A-I & $-1.304 \pm 0.072$ & $0.08983 \pm 0.01305$ \\
\hline 1A-II & $-1.322 \pm 0.042$ & $0.1104 \pm 0.0134$ \\
\hline 1B-I & $-1.250 \pm 0.084$ & $0.1047 \pm 0.01840$ \\
\hline 1B-II & $-1.304 \pm 0.130$ & $0.1622 \pm 0.0244$ \\
\hline
\end{tabular}

\subsubsection{Sloped Ground.}

The sagittal and frontal plane ankle angles during a single trial for the PAFD in powered and passive modes, both with and without the FSS, and under sloped-ground conditions (studies 2A and 2B) are presented in Fig. 16 and Fig. 17, respectively. Mean maximums of these values across several trials are also presented in Table 4. Figure 16 shows that the sagittal plane motion of the PAFD under sloped-ground conditions, is near identical to the motion under level-ground conditions as illustrated in Fig. 15. Figure 17 shows the frontal plane motion of the PAFD. This motion appears to be independent of the power mode and the use of the FSS. This is expected, as the frontal plane system (FPS system) is separated from the sagittal plane system (SEA and FSS), and is thus not affected by the motion or impedance in that is provided by the latter.

At the beginning of the stance phase, the ankle rotates such that the foot is parallel with the slopes surface, producing a frontal ankle angle of $\theta_{F} \approx 20^{\circ}$. The ankle remains rotated for the entirety of the stance phase $(0 \%-60 \%)$, returning rapidly to the default angle at the start of the SW phase. This displays the PAFD's ability to adapt to non-level-ground terrain; maintaining inversion/eversion such that the foot is parallel with a sloped surface, while providing sagittal plane motion identical to that provided under level-ground conditions.

\subsection{Ankle Torques}

\subsubsection{Level Ground.}

The sagittal plane ankle torque during a single trial for the PAFD in powered and passive modes, both with and without the FSS, and under level-ground conditions (studies 1A and 1B) are presented in Fig. 18. Mean maximums of these values across several trials are also presented in Table 5. 


\begin{tabular}{lcc}
\hline Study & Max. plantarflexion torque $(\mathrm{Nm} / \mathrm{kg}) \pm \mathrm{SD}$ & Max. dorsiflexion torque $(\mathrm{Nm} / \mathrm{kg}) \pm \mathrm{SD}$ \\
\hline 2A-I & $-1.307 \pm 0.124$ & $0.1449 \pm 0.0552$ \\
\hline 2A-II & $-0.7082 \pm 0.0468$ & - \\
\hline 2B-I & $-1.395 \pm 0.130$ & - \\
\hline 2B-II & $-0.6449 \pm 0.0954$ & - \\
\hline
\end{tabular}

Table 7. Summary of ankle net work taken from several trials for Session 1.

\begin{tabular}{lc}
\hline Study & Net Work $\left(\mathrm{Jkg}^{-1} \pm\right)$ SD \\
\hline 1A-I & $0.2141 \pm 0.0405$ \\
\hline 1A-II & $0.2770 \pm 0.0344$ \\
\hline 1B-I & $-0.0943 \pm 0.0120$ \\
\hline 1B-II & $-0.0464 \pm 0.0080$ \\
\hline
\end{tabular}

Figure 18 shows the sagittal plane ankle torque of the PAFD. Similar to the frontal plane motion, the sagittal plane torque appears to be independent of the use of the FSS. This is unexpected, although the reason for this may again be explained again by the method employed to manually walk the PAFD. As sagittal plane ankle torque is provided by the force exerted by the HSS and FSS, it is function of their deflection, and as their deflection is a function of the sagittal plane ankle angle, it is therefore a function of the ankle angle. Therefore, the FSS apparent lack of impact on the ankle torque is a consequence of the maximum dorsiflexion without the FSS being much greater than with (discussed in Section 5.1.1), thus increasing the torque of the PAFD without the FSS.

The dorsiflexion torque during $\mathrm{CP}$ for both powered and passive modes appears to follow a near identical path as that of the biological ankle, peaking by approximately the same amount $\left(T \approx 0.1 \mathrm{NmKg}^{-1}\right)$. The same cannot be said for the plantarflexion torque during the rest of the cycle. Under powered mode, plantarflexion torque increases more rapidly than with the biological ankle, and remains near the peak torque for longer. Conversely, under passive mode, plantarflexion toque increases at a near identical rate as the biological ankle, though, similar to powered mode, peak torque is maintained for longer than the biological ankle. The peak plantarflexion torque for both powered and passive modes are near identical $\left(T \approx-1.4 \mathrm{NmKg}^{-1}\right)$, and both are slightly greater than that of the biological ankle $\left(T \approx-1.3 \mathrm{NmKg}^{-1}\right)$.

\subsubsection{Sloped Ground.}

The sagittal plane ankle torque during a single trial for the PAFD in powered and passive modes, both with and without the FSS, and under sloped-ground conditions (studies 2A and 2B) is presented in Fig. 19. Mean maximums of these values across several trials are also presented in Table 6 .

There are significant differences to the torque observed during the Level-Ground Session (studies 1A and 1B), as shown in Fig. 19. Firstly, for all sloped-ground studies (2A and 2B) with the exception of 2A-I no dorsiflexion torque is experienced during CP. It was concluded that this is a result of an experimental setup fault involving the slope's placement onto the forceplate, as the ankle kinematics (see Fig. 16) during these studies appears to be as expected, and thus should be producing torque profiles similar to those for the Level-Ground Session (see Fig. 18). Secondly, the maximum torque exerted is highly dependent on the use of the FSS; maximum torque is greater with the FSS than without. Although this differs from what was observed under level-ground conditions, this is the expected effect of using the FSS.

\subsection{Ankle Net Work}

\subsubsection{Level Ground.}

The sagittal plane ankle torque versus ankle angle during a single trial for the PAFD in powered and passive modes, both with and without the FSS, and under level-ground conditions (studies 1A and 1B) are presented in Fig. 20. Net work values were calculated using MATLAB's trapezoidal numerical integration function, trapz. Mean net work values across several trials are also presented in Table 7.

There are significant and expected differences between the net work under powered and passive modes, as seen in Table 7. Firstly, and most notably, the net work in powered mode is positive, whereas in passive mode it is negative. This is 
Table 8. Summary of ankle net work taken from several trials for Session 2.

\begin{tabular}{lc}
\hline Study & Net Work $\left(\mathrm{Jkg}^{-1} \pm\right) \mathrm{SD}$ \\
\hline 2A-I & $0.1825 \pm 0.0409$ \\
\hline 2A-II & $0.1694 \pm 0.0295$ \\
\hline 2B-I & $-0.0592 \pm 0.0144$ \\
\hline 2B-II & $-0.0303 \pm 0.0111$ \\
\hline
\end{tabular}

a consequence of using the linear actuator; the actuator does work during each cycle by providing significant amounts of plantarflexion torque for PP. Therefore, as the PAFD can provide net-positive work, it thus has the potential to reduce the metabolic rate of the user relative to the use of a passive PAFD (according to findings by [28]). Secondly, the magnitude of net work done while in powered mode is significantly greater than in passive mode; a result of the relatively high amounts of flexion (plantarflexion) torque experienced while in powered mode, and the relatively low amount of flexion (dorsiflexion) torque experienced in passive mode.

\subsubsection{Sloped Ground.}

The sagittal plane ankle torque against ankle angle during a single trial for the PAFD in powered and passive modes, both with and without the FSS, and under sloped-ground conditions for studies 2A and 2B are illustrated in Fig. 21. Similarly, the net work values were calculated using MATLAB's trapz function. The mean net work values across a number of trials are provided in Table 8 .

Similar to under level-ground conditions, there are notable differences between the net work under the powered and passive modes. Under powered mode, net work is positive, whereas under the passive mode it is negative. Additionally, the magnitude of net work done under powered mode is significantly greater than that in passive mode.

\section{Discussions}

The experimental results from the eight studies detailed in Section 5 thoroughly demonstrate the PAFDs abilities and performance, in terms of kinematics, ankle torque, and net work done during a walking gait cycle. These results show that the majority of the design aims and specifications detailed in Section 2 have been met with the PAFD, and some have even been surpassed significantly.

Powered Capabilities. Through the inclusion of a linear actuator, powered ankle motion is provided by the PAFD, allowing for the close emulation of its biological counterpart. This ability is demonstrated by the measurements of ankle angle and torque in Sections 5.1 and 5.2 respectively, showing that the ankle is able to closely follow the motion and torque of a biological ankle during walking. The PAFD is able to correctly plantarflex during CP and PP, and dorsiflex during CD and SW, following closely the ankle angle of a biological ankle. The maximum plantarflexion experienced by the PAFD during walking is also close to that of a biological ankle, reaching up to $\theta \approx-16.27^{\circ}$ and $\theta \approx-14.47^{\circ}$ under level and slopedground conditions respectively. Unfortunately, the same may not be said for the maximum dorsiflexion experienced, which reaches up to $\theta \approx 3.09^{\circ}$ and $\theta \approx 3.54^{\circ}$ under level and sloped-ground conditions respectively. As explained earlier in this section, this is a consequence of the experimental method employed to manually walk the PAFD, and does not demonstrate the maximum dorsiflexion capable by the PAFD. Similar to plantarflexion, the maximum torque experienced is also similar to that of a biological ankle, reaching up to $T \approx 1.32 \mathrm{Nmkg}^{-1}$ and $T \approx 1.31 \mathrm{Nmkg}^{-1}$ under level and sloped-ground conditions.

Not only does the inclusion of the linear actuator allow the ankle to move naturally under the powered mode, but it also provides net-positive work during locomotion. This means that the PAFD has the potential to reduce the metabolic rate of the user. To quantify the work done by the PAFD, the average net work done during several trails was calculated. It was found that the PAFD provides $0.2142 \mathrm{Jkg}^{-1}$ and $0.1825 \mathrm{Jkg}^{-1}$ per walking gait cycle, under level-ground and sloped-ground conditions respectively; a reasonably high amount of work compared to the $\sim 0.13 \mathrm{Jkg}^{-1}$ produced by a biological ankle during walking.

Passive Capabilities. In addition to acting in a powered manner, the PAFD's ability to work as passive prosthesis has also been successfully demonstrated. By disabling the power-supplies, it was shown that the PAFD is able to dorsiflex during $\mathrm{CD}$, akin to a standard passive prosthesis; reaching up to $\theta \approx 9.45^{\circ}$ and $\theta \approx 10.25^{\circ}$ under level and sloped-ground conditions respectively. Although there is no actuator motion to extend the HSS, adequate plantarflexion torque is also provided in the passive mode, reaching up to $T \approx 1.30 \mathrm{Nm} \mathrm{kg}^{-1}$ and $T \approx 1.40 \mathrm{Nmkg}^{-1}$ under level and sloped-ground conditions respectively.

FSS Impact. The use of the FSS has stark effects on the ankle kinematic and torque results. Under both powered and passive modes, and level and sloped-ground conditions, increased dorsiflexion during CD is experienced without the 
Copyright FSS. Unfortunateiy, these results are yet another consequence of the experimental method employed. As the FSS provides additional rotational impedance, more force is required to rotate the PAFD by the operator. Therefore, due to the difficulty to reach the higher, more desirable amounts of dorsiflexion $\left(\theta \approx 10^{\circ}\right)$ when using the FSS, lower dorsiflexion is experienced while using the FSS than without. This may also explain why the ankle torque with the FSS is near identical to without, as the ankle torque is a function of the ankle angle.

2-DOF Capabilities. Through the use of a custom U-Joint ankle in conjunction with a linear actuator the PAFD is able to provide powered flexion and 2-DOF at the ankle joint; the PAFD provides powered and passive motion in the sagittal plane, and passive motion in the frontal plane. This poses the potential for increased terrain adaption, and a more natural gait compared to the standard 1-DOF powered PAFDs currently used.

To demonstrate the PAFD's powered 2-DOF capabilities, sloped-ground walking trials were undertaken. The PAFD's sagittal plane motion (plantarflexion/dorsiflexion) was found to be unaffected by any extent of frontal plane motion (inversion/eversion), performing equally well under sloped and level-ground conditions. The sloped-ground session also demonstrated PAFD's ability to invert/evert in such a way as to allow the sole of the foot to become parallel with the sloped surface, adapting to the terrain; the ankle inverted/everted $\sim 20^{\circ}$ to a surface of slope $20^{\circ}$. The response of the PAFD to the sloped surface was also relatively fast, taking $\sim 10 \%$ of the cycle to adapt to the presented terrain's surface after HS, and return to the default frontal plane position $\left(\theta_{F}=0^{\circ}\right)$ after TO. Although this response performance is satisfactory, especially when considering the exclusively passive nature of the frontal plane system, a faster response would be desirable. The PAFD's frontal plane motion was also found to be independent of the power mode used (powered or passive), meaning that the 2-DOF capabilities of the device do not adversely affect its passive performance.

\section{Conclusions}

This paper has detailed the design and testing of a novel powered PAFD which provides 2-DOF motion at the ankle. The proposed PAFD's innovation arises primarily from the design of the ankle itself. By designing a custom U-Joint ankle, frontal plane motion is permitted without any sacrifice to powered sagittal plane motion, thus providing powered sagittal plane flexion and 2-DOF at the ankle. Sagittal plane flexion utilizes a SEA to emulate the biomechanics of the calf muscle and Achilles tendon, and acts in parallel with a spring system (FSS). Frontal plane flexion is completely passive, relying only on a parallel spring system (FPS system) to provide inversion/eversion impedance.

The experiments conducted have proved to be highly successful. A total of eight studies were undertaken, providing sufficient data to characterize the PAFD's sagittal and frontal plane performance. It was shown that the PAFD's powered sagittal plane kinematics are independent of the ground conditions, performing equally well under level and sloped-ground conditions. The sagittal plane ankle angle followed closely the motion of a biological ankle, correctly undergoing plantarflexion during $\mathrm{CP}$ and PP, and dorsiflexion during CD and SW. The primary difference between the achieved PAFD motion and that of a biological ankle was the extent of dorsiflexion during $\mathrm{CD}$, although this may be explained due to experimental methods employed. Passive capabilities were also shown. Similar to the powered mode, passive sagittal kinematics were found to be independent of the ground conditions, performing close to the target motion under both level and sloped-ground conditions. The powered kinematic results also show the robustness of the control system; the pressure sensor's ability to detect gait events, the IR range sensor's ability to measure actuator length, and the finite state controller's ability to process all sensor data as intended and in real-time.

Similar to ankle kinematics, the ankle torque was also found to be independent of the ground conditions. Under both level and sloped-ground conditions, maximum ankle torque reached or surpassed the biological target. Net-positive work is also done by the PAFD, providing the potential for it to reduce user metabolic rate.

The PAFD's 2-DOF abilities were successfully demonstrated by the sloped-ground session; the PAFD demonstrated the capacity to rapidly adapt to terrain by inverting/everting to the sloped conditions, while simultaneously providing powered sagittal plane motion. The ankle inverted/everted $\sim 20^{\circ}$ to a slope of angle $20^{\circ}$, and took $\sim 10 \%$ of the gait cycle to respond to the presented ground conditions. The frontal plane motion was also found to be independent of the power mode used, meaning that the PAFD was able to adapt to the sloped conditions equally well under the powered and passive modes.

\section{References}

[1] Mai, A., and Commuri, S., 2016. "Intelligent control of a prosthetic ankle joint using gait recognition". Control Engineering Practice, 49, pp. 1-13.

[2] Mak, A.-T., Zhang, M., and Leung, A.-L., 2003. “Artificial limbs”. Comprehensive Structural Integrity, 9, pp. 329-363.

[3] Walker, C., Ingram, R., Hullin, M., and McCreath, S., 1994. "Lower limb amputation following injury: a survey of long-term functional outcome". Injury, 25(6), pp. 387-392.

[4] Thurston, A., 2007. "Pare and prosthetics: The early history of artificial limbs". ANZ Journal of Surgery, 2007(77), pp. 1114-1119. 
Copyright ${ }^{2}[5]$ Ron, S., 2002 . Prosthetics and Orthotics: Lower limb and Spinal. Lippincott Williams \& Wilkins, Baltimore, Maryland, USA.

[6] Collins, S., and Kuo, A., 2003. "Controlled energy storage and return prosthesis reduces metabolic cost of walking". In ISB XXth Congress and the American Society of Biomechanics Annual Meeting, p. 804.

[7] Li, C., Tokuda, M., Hashimoto, Y., Morimoto, S., Nakagawa, A., and Akazawa, Y., 2006. "Research and development of the intelligently-controlled prosthetic ankle joint". In 2006 International Conference on Mechatronics and Automation, pp. 1114-1119.

[8] Eilenberg, M., Geyer, H., and Herr, H., 2010. "Control of a powered ankle-foot prostheses based on a neuromuscular model". IEEE Transactions on Neural Systems and Rehabilitation Engineering, 18(2), pp. 164-173.

[9] Grabowski, A., and Susan, D., 2013. "Effects of a powered ankle-foot prosthesis on kinetic loading of the unaffected leg during level-ground walking". Journal of Neuroengineering and Rehabilitation, 10, p. 49.

[10] Herr, H., and Grabowski, A., 2012. "Bionic ankle-foot prostheses normalizes walking gait for persons with leg amputation". Proceeding of the Royal Society B: Biological Sciences, 279(1728), pp. 457-464.

[11] Genin, J. J., Bastien, G. J., Franck, B., Detrembleur, C., and Willems, P. A., 2008. "Effect of speed on the energy cost of walking in unilateral traumatic lower limb amputees". European Journal of Applied Physiology, 103, pp. 655-663.

[12] Houdijk, H., Pollmann, E., Groenewold, M., Wiggerts, H., and Polomski, W., 2009. "The energy cost for the step-tostep transition in amputee walking”. Gait Posture, 30, pp. 35-40.

[13] Hsu, M., Nielsen, D., Lin-Chan, S., and Shurr, D., 2006. "The effects of prosthetic foot design on physiologic measurements, self-selected walking velocity, and physical activity in people with transtibial amputation". Archives of Physical Medicine and Rehabilitation, 87(1), pp. 123-129.

[14] Sanderson, D., and Martin, P., 1997. "Lower extremity kinematic and kinetic adaptations in unilateral below-knee amputees during walking". Gait Posture, 6(2), pp. 126-136.

[15] Au, S. K., and Herr, H. M., 2008. "Powered ankle-foot prosthesis". IEEE Robotics \& Automation Magazine, 15(3), pp. 52-59.

[16] Au, S., Bonato, P., and Herr, H., 2005. "An emg-position controlled system for an active ankle-foot prosthesis: An initial experimental study". In Proceedings of the 2005 IEEE 9th International Conference on Rehabilitation Robotics, pp. $375-379$.

[17] Au, S., Dilworth, P., and Herr, H., 2006. "An ankle-foot emulation system for the study or human walking biomechanics". In Proceedings of the 2006 IEEE International Conference on Robotics and Automation, pp. 2939-2945.

[18] Au, S. K., Herr, H., Weber, J., and Martinez-Villalpando, E. C., 2007. "Powered ankle-foot prosthesis for the improvement of amputee ambulation". In Proceedings of the 29th Annual International Conference of the IEEE EMBS, pp. 3020-3026.

[19] Au, S. andBerniker, M., and Herr, H., 2008. "Powered ankle-foot prosthesis to assist level-ground and stair-descent gaits". Neural Networks, 21, pp. 654-666.

[20] Au, S., Weber, J., and Herr, H., 2009. "Powered ankle-foot prosthesis improves walking metabolic economy". IEEE Transactions on Robotics, 25(1), pp. 51-66.

[21] Li, C., Morimot, S., Furusho, J., and Kikuch, T., 2009. "Development of a prosthetic ankle-foot and its slope-walking experiments". In Proceedings of the 2009 IEEE International Conference on Mechatronics and Automation, pp. 18061810.

[22] Michael, J., 1992. "Prosthetic feet: Opinions for the older client". Topics in Geriatric Rehabilitation, 8(1), pp. 30-38.

[23] Whittle, M., 2007. Gait Analysis: An Introduction. Butterworth-Heinemann, Jordan Hill, Oxford, UK.

[24] Palmer, M. L., 1999. "Sagittal plane characterization of normal human ankle function across a range of walking gait speeds". Msc thesis, University of Utah, USA.

[25] Hitt, J., Oymagil, A. M., Sugar, T., Hollander, K., Boehler, A., and Fleeger, J., 2007. "Dynamically controlled anklefoot orthosis (dco) with regenerative kinetics: Incrementally attaining user portability". In Proceedings 2007 IEEE International Conference on Robotics and Automation, pp. 1541-1546.

[26] Moriguchi, C., Sato, T., and Coury, H. G., 2007. "Ankle movements during normal gait evaluated by flexible electrogoniometer". Brazilian Journal of Physical Therapy, 11, pp. 205-211.

[27] Plocharski, M., and Plocharski, P., 2013. "Ankle joint stiffness during phases of human walking". Master thesis, Aalborg University, Denmark.

[28] Au, S. K.-W., 2007. "Powered ankle-foot prosthesis for the improvement of amputee walking economy". Phd thesis, Massachusetts Institute of Technology, USA. 
(a)

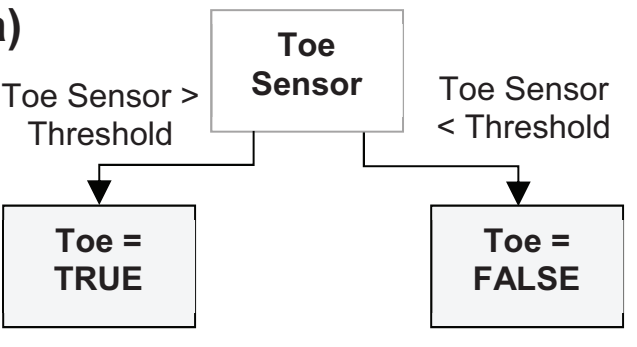

(b)

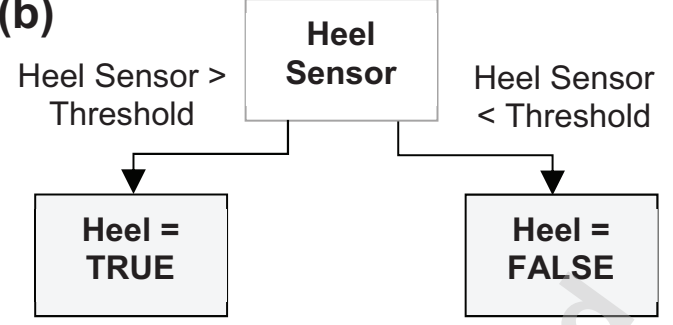

(c)



(d)

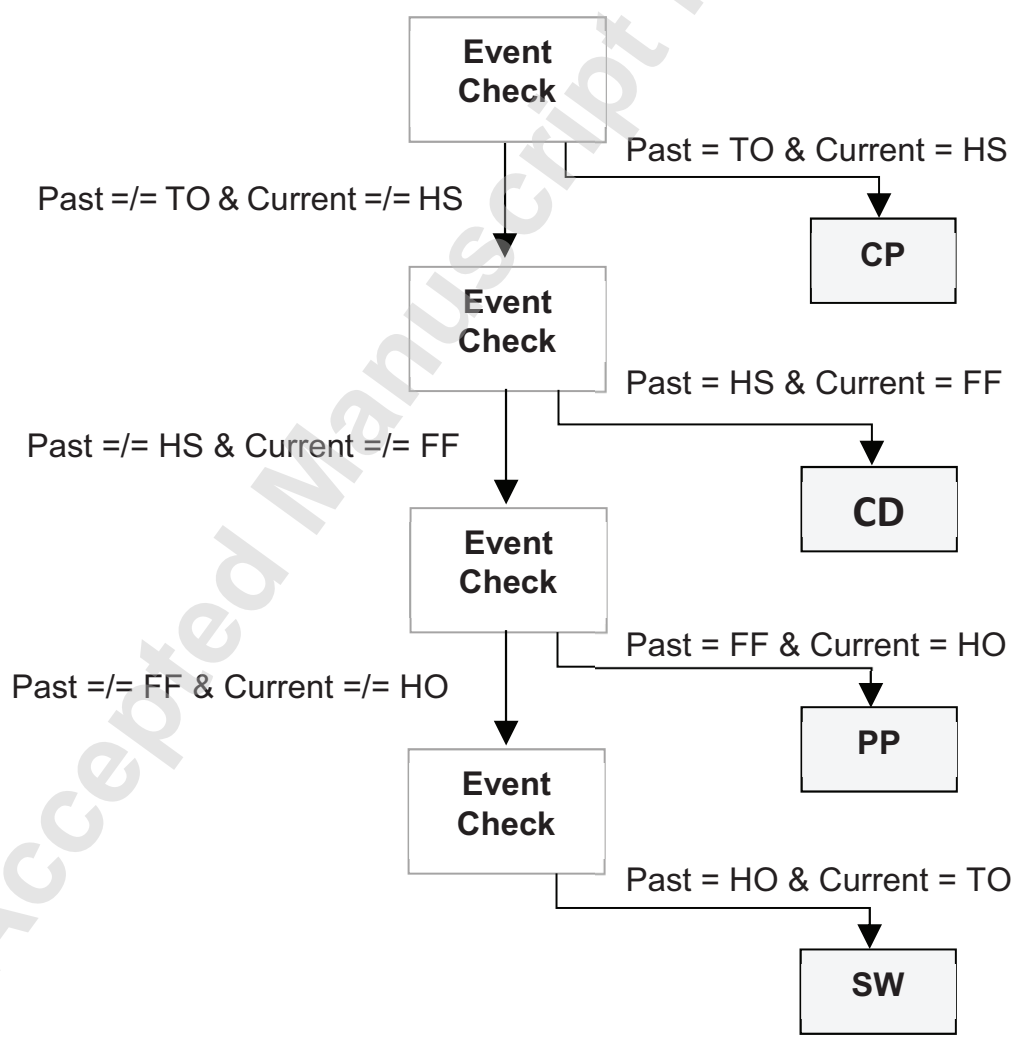

Fig. 8. Classification trees for the determination of: (a) prosthetic toe; (b) heel states; (c) gait events by utilising the toe and heel states, $T R U E$ means there is contact with the ground, whereas FALSE means there is no contact; and (d) the current gait sub-phase. 


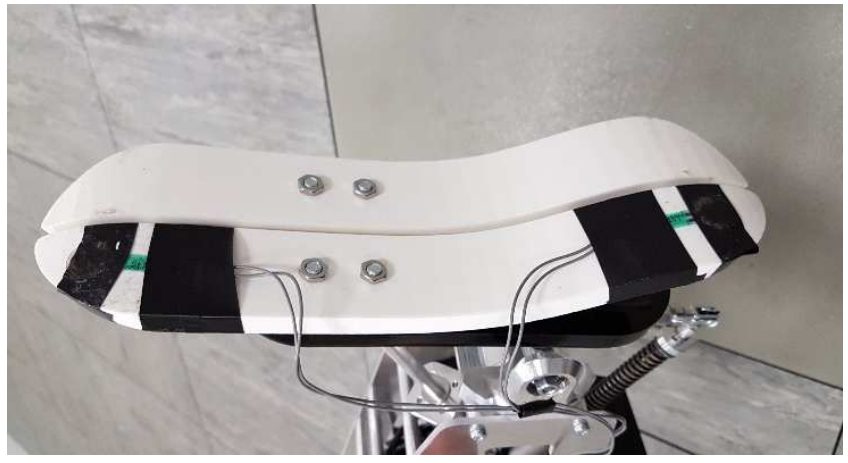

Fig. 9. Heel and toe pressure sensors.

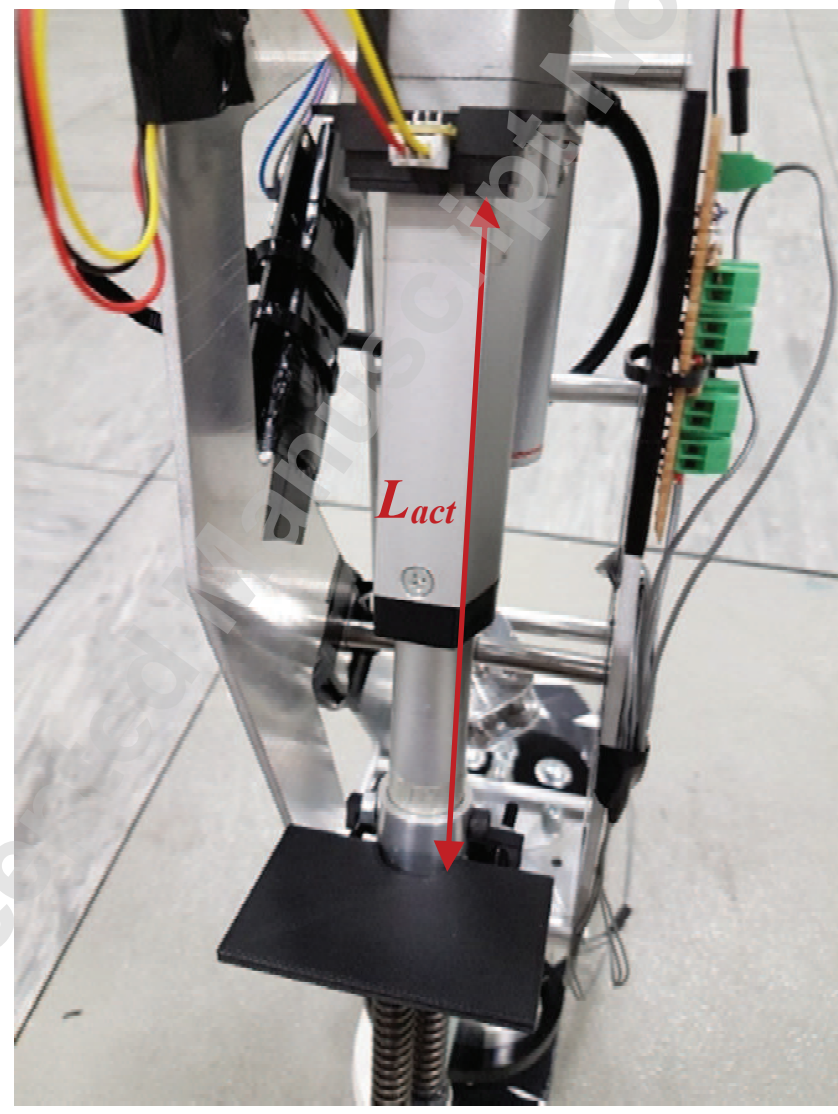

Fig. 10. The actuator sensor setup, showing the IR range sensor, and reflective panel. The actuator length, $L_{a t c}$, is taken as the distance between the IR sensor and the reflective panel. 


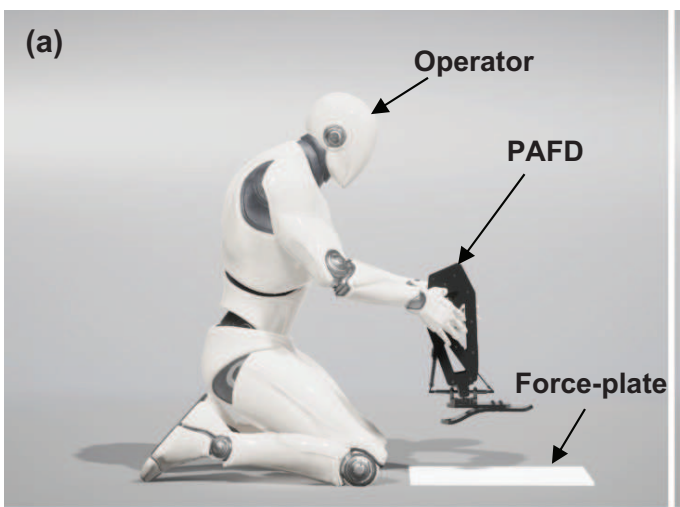

(b)

(c)

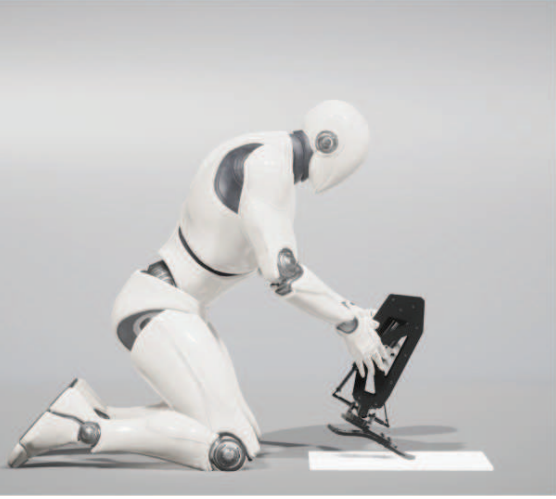

(d)

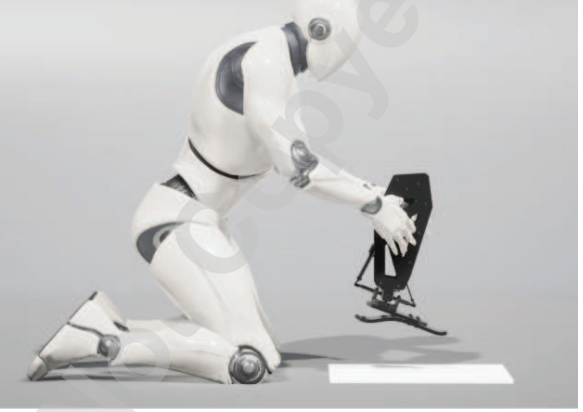

Fig. 11. Setup used to emulate the motion of the lower limb during a walking gait cycle: (a) PAFD lowered to the ground for HS, (b) CP and CD takes place as the operator applies their weight onto the PAFD, (c) PP causes the PAFD to lift the weight of the operator and (d) the PAFD is lifted from the ground for HO. This setup allowed for a large amount of force to be consistently applied across all trials.
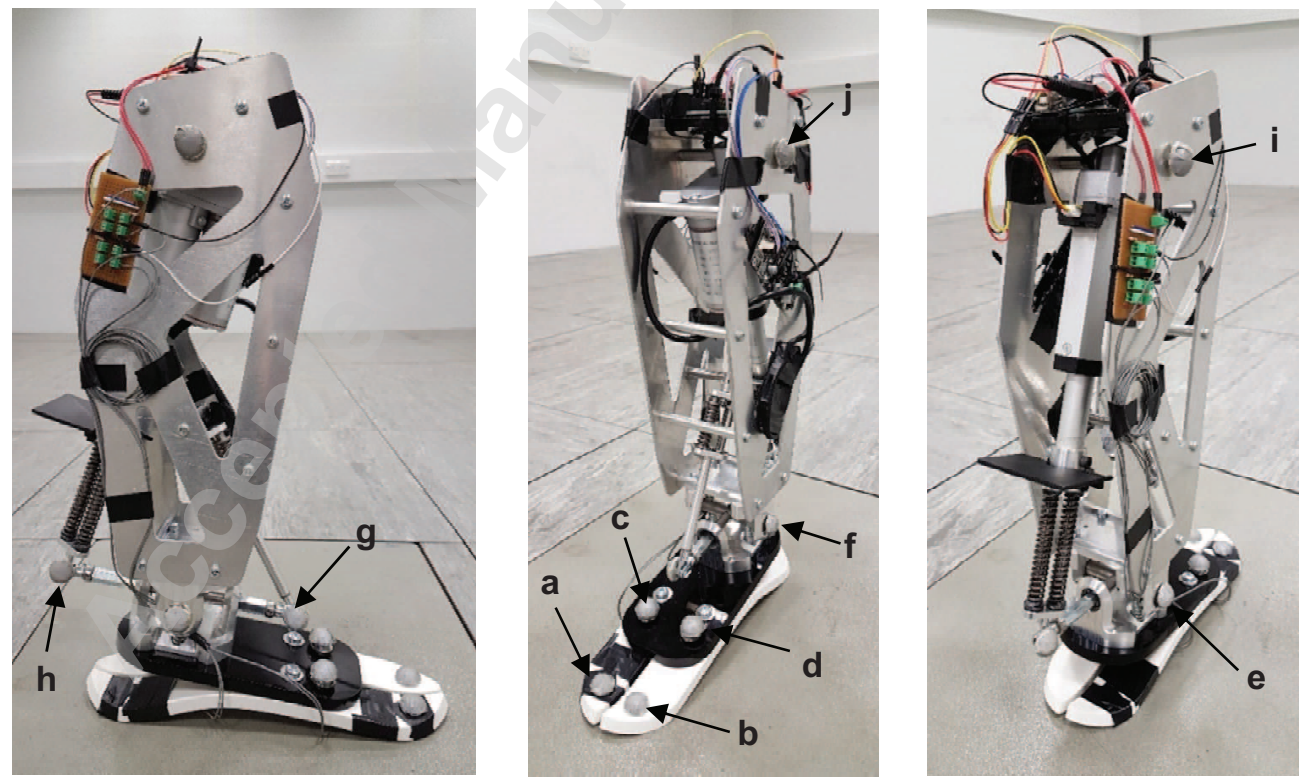

Fig. 12. Experimental setup of the PAFD. The positions of the IR markers were: a) right toe 1, b) left toe 1, (c) right toe 2, d) left toe 2, e) ankle right, f) ankle left, g) ankle forward, h) ankle back, i) shank right, and j) shank left. 

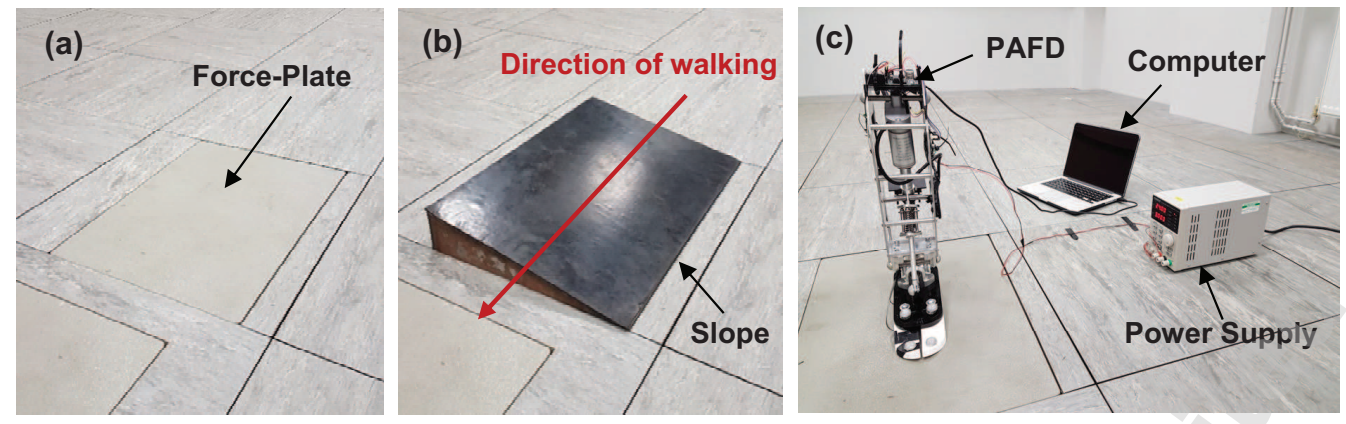

Fig. 13. The force-plate setup for (a) Session 1 and (b) Session 2, and (c) the general setup for all experiments.

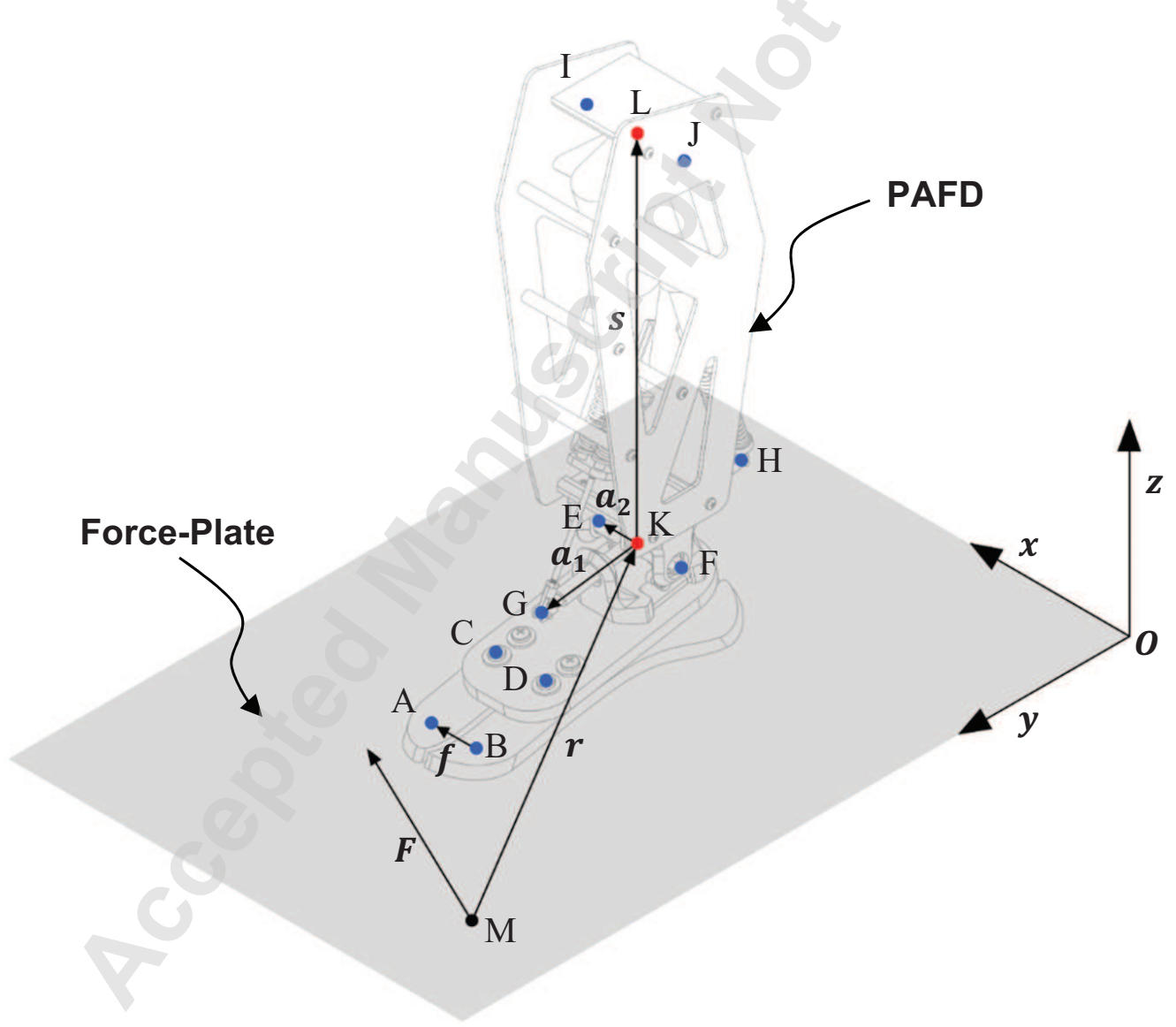

Fig. 14. Graphical representation of the vectors used to calculate ankle angle and ankle torque. Points $\mathrm{A}$ to $\mathrm{J}$ represent the captured marker positions, and points $\mathrm{K}$ and $\mathrm{L}$ represent calculated positions. 


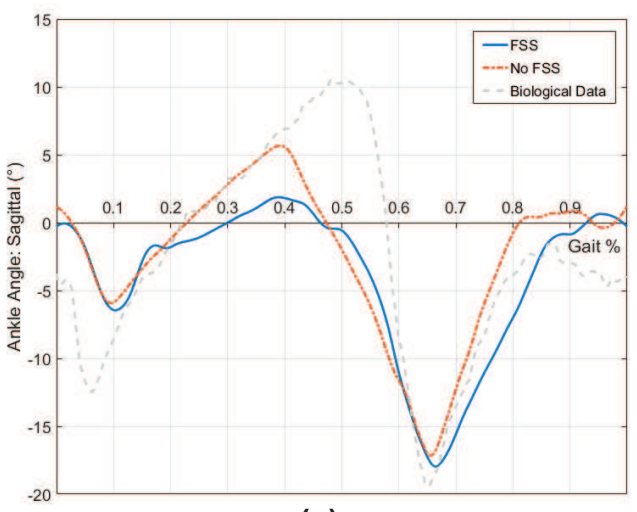

(a)

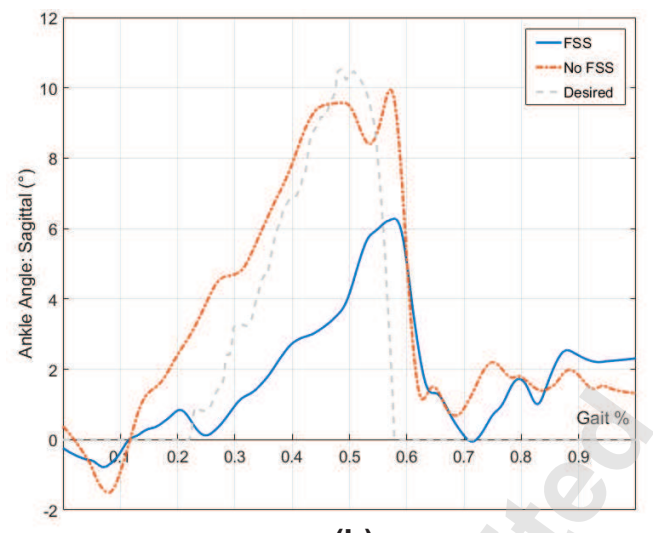

(b)

Fig. 15. Ankle angle in the sagittal plane for a single trial from studies $1 \mathrm{~A}-\mathrm{I}$ and $1 \mathrm{~A}-\mathrm{II}$ (a); and 1B-I and 1B-II (b). The time period for the shown trails are: (a) 7.07s and 7.61s for 'FSS' and 'no FSS' respectively; b) $5.1 \mathrm{~s}$ and 4.45s for 'FSS' and 'no FSS' respectively. For comparison, the desired ankle angle using data modified from [24] is also displayed.

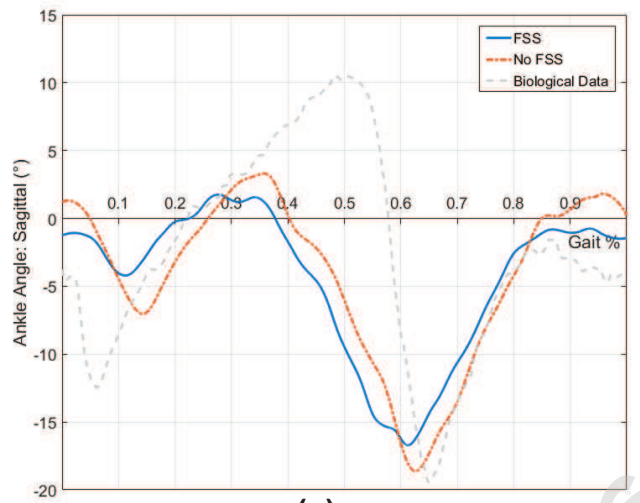

(a)

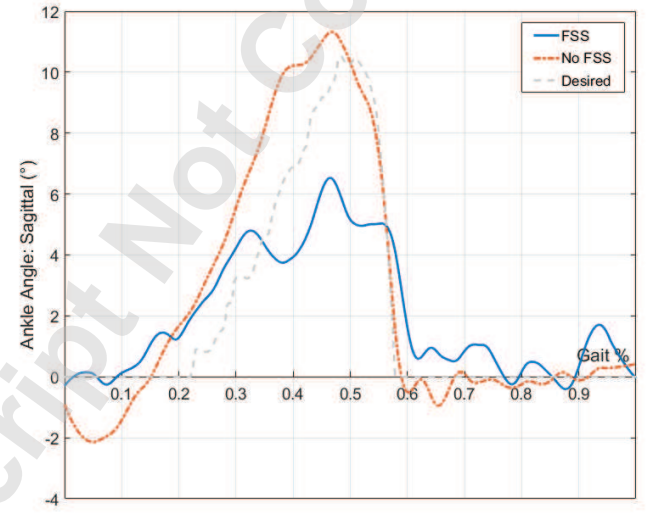

(b)

Fig. 16. Ankle angle in the sagittal plane for a single trial from studies 2A-I and 2A-II (a); and 2B-I and 2B-II (b). The time period for the shown trails are: (a) 6.65s and 6.52s for 'FSS' and 'no FSS' respectively; (b) 4.39s and 3.51s for 'FSS' and 'no FSS' respectively. For comparison, the desired ankle angle using data modified from [24] is also displayed.

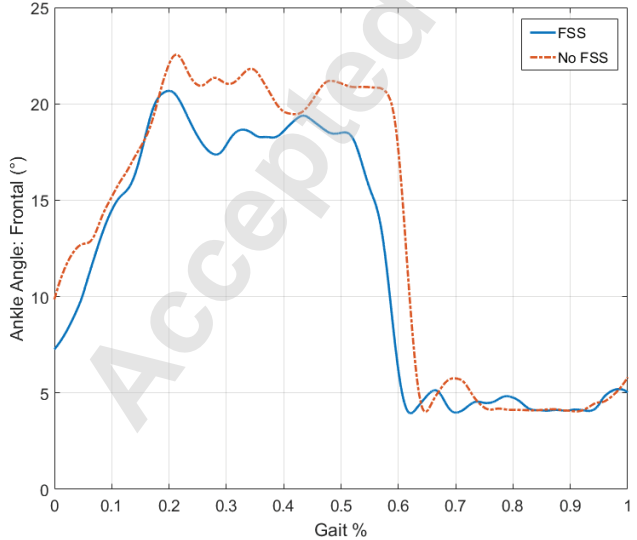

(a)

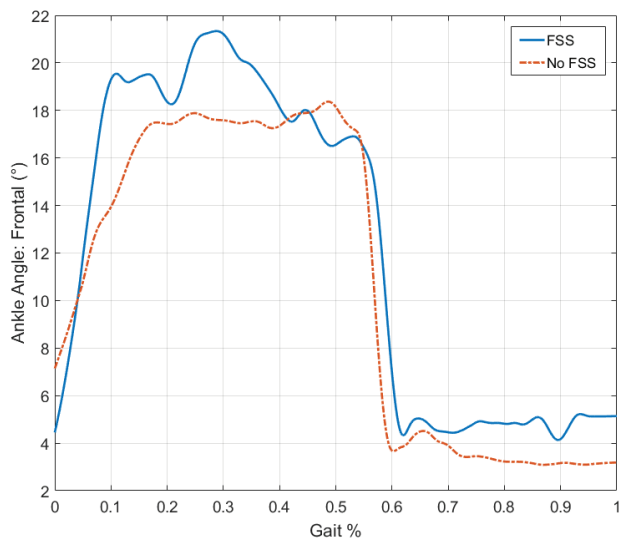

(b)

Fig. 17. Ankle angle in the frontal plane for a single trial from studies $2 \mathrm{~A}-\mathrm{I}$ and $2 \mathrm{~A}-\mathrm{II}$ (a); and 2B-I and 2B-II (b). The time period for the shown trails are: (a) 6.65s and 6.52s for 'FSS' and 'no FSS' respectively; (b) 4.39s and 3.51s for 'FSS' and 'no FSS' respectively. 


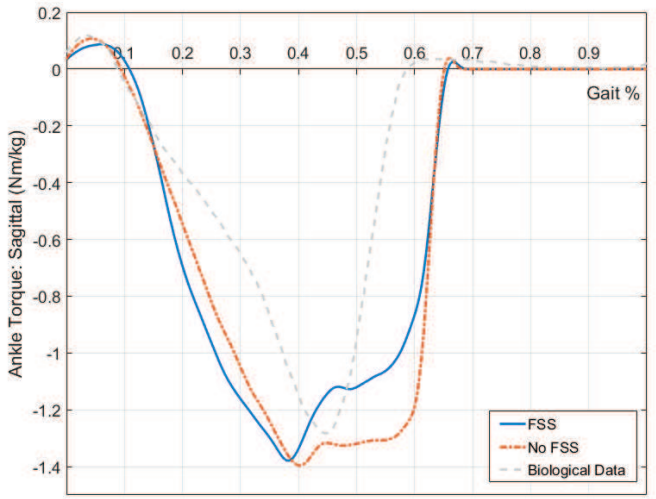

(a)

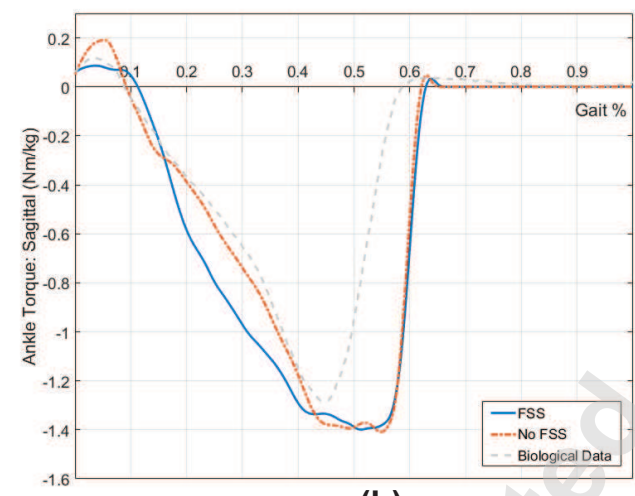

(b)

Fig. 18. Ankle torque in the sagittal plane for a single trial from studies $1 \mathrm{~A}-\mathrm{I}$ and $1 \mathrm{~A}-\mathrm{II}$ (a); and 1B-I and 1B-II (b). The time period for the shown trails are: (a) 7.07s and 7.61s for 'FSS' and 'no FSS' respectively; b) $5.1 \mathrm{~s}$ and $4.45 \mathrm{~s}$ for 'FSS' and 'no FSS' respectively. For comparison, biological ankle torque data from [23] is also displayed.

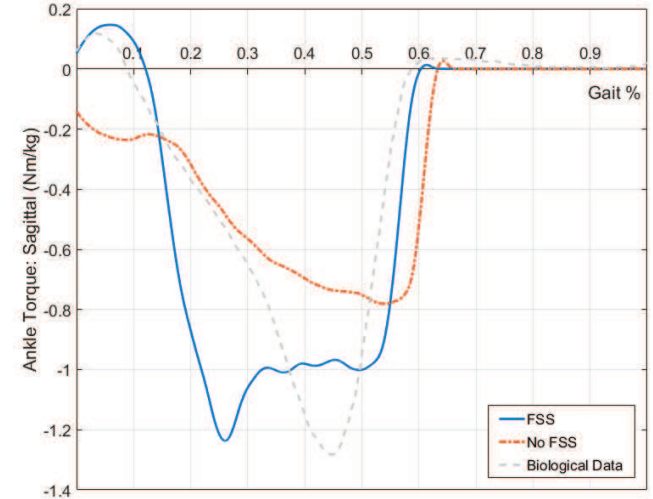

(a)

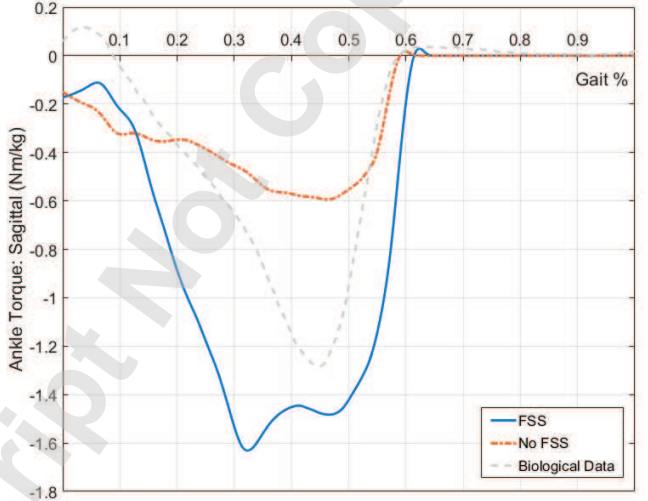

(b)

Fig. 19. Ankle torque in the sagittal plane for a single trial from studies $2 \mathrm{~A}-\mathrm{I}$ and $2 \mathrm{~A}-\mathrm{II}$ (a); and $2 \mathrm{~B}-\mathrm{I}$ and $2 \mathrm{~B}-\mathrm{II}$ (b). The time period for the shown trails are: (a) 6.65s and 6.52s for 'FSS' and 'no FSS' respectively; (b) 4.39s and 3.51s for 'FSS' and 'no FSS' respectively. For comparison, biological ankle torque data from [23] is also displayed.

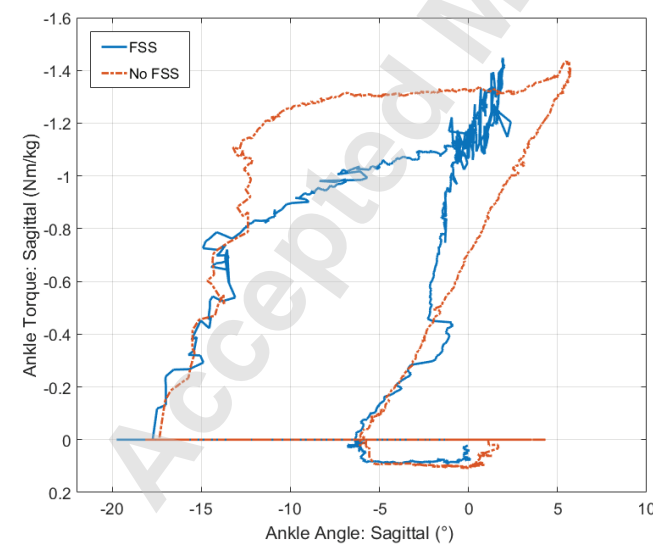

(a)

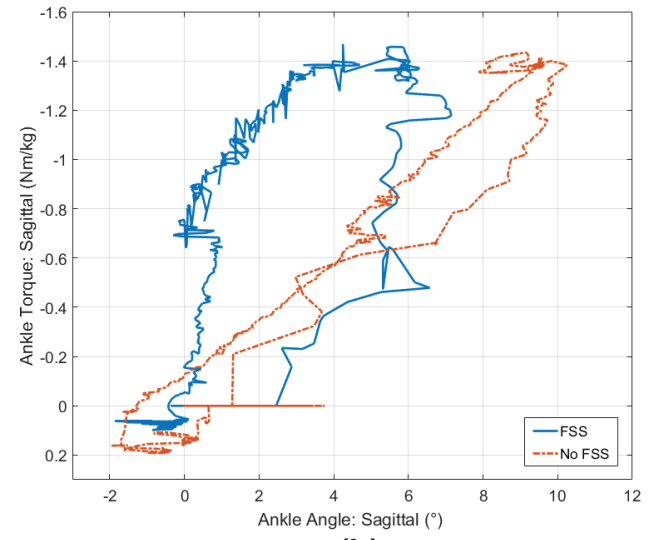

(b)

Fig. 20. Ankle torque against ankle angle in the sagittal plane for a single trial from studies $1 \mathrm{~A}-\mathrm{I}$ and $1 \mathrm{~A}-\mathrm{II}$ (a); and 1B-I and 1B-II (b). By calculating the area enclosed within each plot, the net work is calculated. Powered net work: $0.1976 \mathrm{Jkg}^{-1}$ (FSS), and 0.302 Jkg ${ }^{-1}$ (No FSS). Passive net work: $-0.0961 \mathrm{Jkg}^{-1}$ (FSS), and $-0.0426 \mathrm{Jkg}^{-1}$ (No FSS). The time period for the shown trails are: (a) $7.07 \mathrm{~s}$ and $7.61 \mathrm{~s}$ for 'FSS' and 'no FSS' respectively; b) $5.1 \mathrm{~s}$ and 4.45 s for 'FSS' and 'no FSS' respectively. 


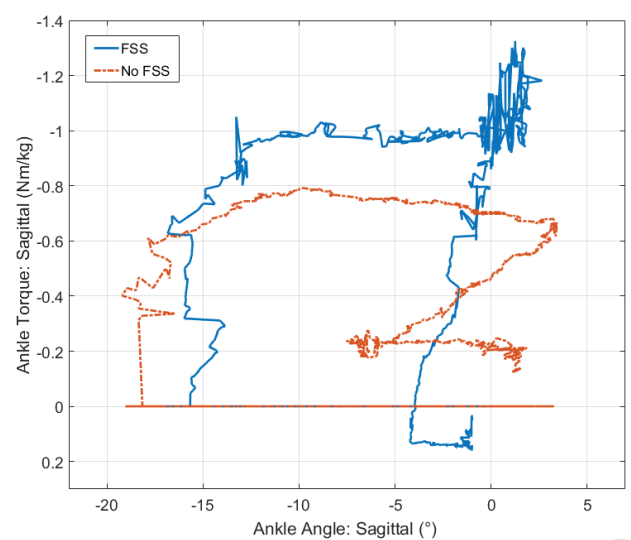

(a)

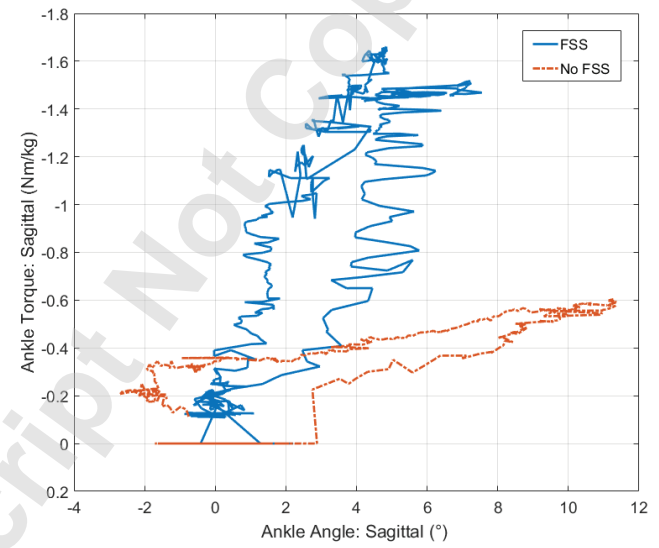

(b)

Fig. 21. Ankle torque against ankle angle in the sagittal plane for a single trial from studies $2 \mathrm{~A}-\mathrm{I}$ and $2 \mathrm{~A}-\mathrm{II}$ (a); and 2B-I and 2B-II (b). By calculating the area enclosed within each plot, the net work is calculated. Powered net work: $0.2150 \mathrm{Jkg}^{-1}$ (FSS), and $0.2285 \mathrm{Jkg}{ }^{-1}$ (No FSS). Passive net work: $-0.0491 \mathrm{Jkg}^{-1}$ (FSS), and -0.0395. $\mathrm{kg}^{-1}$ (No FSS). The time period for the shown trails are: (a) 6.65s and 6.52s for 'FSS' and 'no FSS' respectively; (b) 4.39s and 3.51s for 'FSS' and 'no FSS' respectively. 


\section{List of figure captions}

Fig. 1. (a) CAD render of the final PAFD design, (b) Sagittal plane cut-out diagram, and (b) CAD render of the PAFD being worn by a user.

Fig. 2. Kinematic diagram of the PAFDs: (a) Sagittal plane, and (b) Frontal plane.

Fig. 3. The intended motion of the PAFD in the sagittal (a) and frontal (b) planes. The key gait events, periods, and sub-phases represented are: (1) HS, (2) CD, (3) HO, (4) PP, (5) TO, and (6) SW.

Fig. 4. Desired displacement of the actuator, and SEA. The SEA displacement data was generated using desired ankle angle data from [1]; graph plotted in the style of [25].

Fig. 5. CAD render and photographs of the PAFD (including control system).

Fig. 6. Close up of the U-joint ankle mechanism: a) Forward PDMA, b) U-joint Top, c) Heel PDMA, d) U-joint Base, e) IEMA, and f) FPS.

Fig. 7. The assembled prosthetic foot: a) Ankle platform, b) Right stabilizer, and c) Left stabilizer.

Fig. 8. Classification trees for the determination of: (a) prosthetic toe; (b) heel states; (c) gait events by utilising the toe and heel states, TRUE means there is contact with the ground, whereas FALSE means there is no contact; and (d) the current gait sub-phase.

Fig. 9. Heel and toe pressure sensors.

Fig. 10. The actuator sensor setup, showing the IR range sensor, and reflective panel. The actuator length, $L_{\text {atc, }}$ is taken as the distance between the IR sensor and the reflective panel.

Fig. 11. Setup used to emulate the motion of the lower limb during a walking gait cycle: (a) PAFD lowered to the ground for $\mathrm{HS}$, (b) CP and CD takes place as the operator applies their weight onto the PAFD, (c) PP causes the PAFD to lift the weight of the operator and (d) the PAFD is lifted from the ground for HO. This setup allowed for a large amount of force to be consistently applied across all trials.

Fig. 12. Experimental setup of the PAFD. The positions of the IR markers were: a) right toe $1, b)$ left toe 1 , (c) right toe 2 , d) left toe 2 , e) ankle right, f) ankle left, g) ankle forward, h) ankle back, i) shank right, and j) shank left.

Fig. 13. The force-plate setup for (a) Session 1 and (b) Session 2, and (c) the general setup for all experiments.

Fig. 14. Graphical representation of the vectors used to calculate ankle angle and ankle torque. Points $A$ to $J$ represent the captured marker positions, and points $K$ and $L$ represent calculated positions.

Fig. 15. Ankle angle in the sagittal plane for a single trial from studies $1 \mathrm{~A}-\mathrm{I}$ and $1 \mathrm{~A}-\mathrm{II}$ (a); and $1 \mathrm{~B}-\mathrm{I}$ and 1B-II (b). The time period for the shown trails are: (a) 7.07s and 7.61s for 'FSS' and 'no FSS' respectively; b) $5.1 \mathrm{~s}$ and $4.45 \mathrm{~s}$ for 'FSS' and 'no FSS' respectively. For comparison, the desired ankle angle using data modified from [24] is also displayed.

Fig. 16. Ankle angle in the sagittal plane for a single trial from studies $2 \mathrm{~A}-\mathrm{I}$ and $2 \mathrm{~A}-\mathrm{II}$ (a); and 2B-I and 2B-II (b). The time period for the shown trails are: (a) $6.65 \mathrm{~s}$ and $6.52 \mathrm{~s}$ for 'FSS' and 'no FSS' respectively; (b) 4.39s and 3.51s for 'FSS' and 'no FSS' respectively. For comparison, the desired ankle angle using data modified from [24] is also displayed. 
Fig. 17. Ankle angle in the frontal plane for a single trial from studies $2 \mathrm{~A}-\mathrm{I}$ and $2 \mathrm{~A}-\mathrm{II}$ (a); and $2 \mathrm{~B}-\mathrm{I}$ and 2B-II (b). The time period for the shown trails are: (a) $6.65 \mathrm{~s}$ and $6.52 \mathrm{~s}$ for 'FSS' and 'no FSS' respectively; (b) 4.39s and 3.51s for 'FSS' and 'no FSS' respectively.

Fig. 18. Ankle torque in the sagittal plane for a single trial from studies $1 \mathrm{~A}-\mathrm{I}$ and $1 \mathrm{~A}-\mathrm{II}$ (a); and 1B-I and 1B-II (b). The time period for the shown trails are: (a) 7.07s and 7.61s for 'FSS' and 'no FSS' respectively; b) $5.1 \mathrm{~s}$ and $4.45 \mathrm{~s}$ for 'FSS' and 'no FSS' respectively. For comparison, biological ankle torque data from [23] is also displayed.

Fig. 19. Ankle torque in the sagittal plane for a single trial from studies $2 \mathrm{~A}-\mathrm{I}$ and $2 \mathrm{~A}-\mathrm{II}$ (a); and 2B-I and 2B-II (b). The time period for the shown trails are: (a) 6.65s and 6.52s for 'FSS' and 'no FSS' respectively; (b) $4.39 \mathrm{~s}$ and $3.51 \mathrm{~s}$ for 'FSS' and 'no FSS' respectively. For comparison, biological ankle torque data from [23] is also displayed.

Fig. 20. Ankle torque against ankle angle in the sagittal plane for a single trial from studies $1 \mathrm{~A}-\mathrm{I}$ and 1A-II (a); and 1B-I and 1B-II (b). By calculating the area enclosed within each plot, the net work is calculated. Powered net work: $0.1976 \mathrm{Jkg}-1$ (FSS), and 0.302 Jkg-1(No FSS). Passive net work: $0.0961 \mathrm{Jkg}-1$ (FSS), and $-0.0426 \mathrm{Jkg}-1$ (No FSS). The time period for the shown trails are: (a) 7.07s and 7.61s for 'FSS' and 'no FSS' respectively; b) 5.1s and 4.45s for 'FSS' and 'no FSS' respectively.

Fig. 21. Ankle torque against ankle angle in the sagittal plane for a single trial from studies $2 \mathrm{~A}-\mathrm{I}$ and 2A-II (a); and 2B-I and 2B-II (b). By calculating the area enclosed within each plot, the net work is calculated. Powered net work: $0.2150 \mathrm{Jkg}-1$ (FSS), and $0.2285 \mathrm{Jkg}-1$ (No FSS). Passive net work: $0.0491 \mathrm{Jkg}-1$ (FSS), and $-0.0395 \mathrm{Jkg}-1$ (No FSS). The time period for the shown trails are: (a) $6.65 \mathrm{~s}$ and $6.52 \mathrm{~s}$ for 'FSS' and 'no FSS' respectively; (b) 4.39s and 3.51s for 'FSS' and 'no FSS' respectively.

\section{List of table captions}

Table 1. Design specifications and parameters that will be aimed for.

Table 2. Desired design specifications and what was finally achieved in the prototype.

Table 3. Summary of ankle angle measurements taken from several trials for Session 1. Max. Inversion/Eversion measurements are not presented for Session 1 as all studies are under levelground conditions, and thus negligible amounts of inversion/eversion are undergone.

Table 4. Summary of ankle angle measurements taken from several trials for all Session 2.

Table 5. Summary of ankle torque measurements taken from several trials for Session 1. Max. Inversion/Eversion measurements are not presented for Session 1 all studies are under level-ground conditions, and thus no inversion/eversion is undergone.

Table 6. Summary of ankle torque measurements taken from several trials for Session 2.

Table 7. Summary of ankle net work taken from several trials for Session 1.

Table 8. Summary of ankle net work taken from several trials for Session 2. 